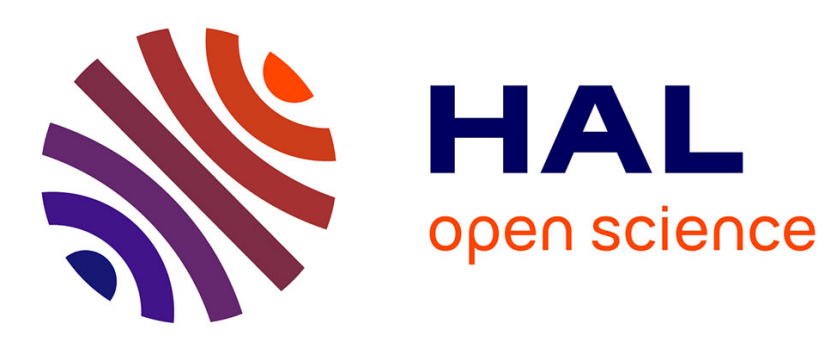

\title{
Robust Inequality of Opportunity Comparisons: Theory and Application to Early Childhood Policy Evaluation
}

Francesco Andreoli, Tarjei Havnes, Arnaud Lefranc

\section{To cite this version:}

Francesco Andreoli, Tarjei Havnes, Arnaud Lefranc. Robust Inequality of Opportunity Comparisons: Theory and Application to Early Childhood Policy Evaluation. Review of Economics and Statistics, 2019, 101 (2), pp.355-369. 10.1162/rest_a_00747. hal-02528191

\section{HAL Id: hal-02528191 \\ https://hal.science/hal-02528191}

Submitted on 1 Apr 2020

HAL is a multi-disciplinary open access archive for the deposit and dissemination of scientific research documents, whether they are published or not. The documents may come from teaching and research institutions in France or abroad, or from public or private research centers.
L'archive ouverte pluridisciplinaire HAL, est destinée au dépôt et à la diffusion de documents scientifiques de niveau recherche, publiés ou non, émanant des établissements d'enseignement et de recherche français ou étrangers, des laboratoires publics ou privés. 


\title{
Robust inequality of opportunity comparisons: Theory and application to early-childhood policy evaluation* ${ }^{* \dagger}$
}

\author{
Francesco Andreoli $^{\mathrm{a}}{ }^{\ddagger}$, Tarjei Havnes $^{\mathrm{b}}$, and Arnaud Lefranc ${ }^{\mathrm{c}}$ \\ ${ }^{\mathrm{a}}$ LISER - Luxembourg Institute of Socio-Economic Research \\ ${ }^{\mathrm{b}}$ University of Oslo \\ ${ }^{\mathrm{c}}$ THEMA, University of Cergy-Pontoise and IZA
}

July 2017

\begin{abstract}
This paper develops a criterion to assess equalization of opportunity that is consistent with theoretical views of equality of opportunity. We characterize inequality of opportunity as a situation where some groups in society enjoy an illegitimate advantage. In this context, equalization of opportunity requires that the extent of the illegitimate advantage enjoyed by the privileged groups falls. Robustness requires that this judgement be supported by the broadest class of individual preferences. We formalize this criterion in a decision theoretic framework, and derive an empirical condition for equalization of opportunity based on observed opportunity distributions. The criterion is used to assess the effectiveness of child care at equalizing opportunity among children, using quantile treatment effects estimates of a major child care reform in Norway. Overall, we find strong evidence supporting equalization of opportunity.
\end{abstract}

Keywords: Equality of opportunity, public policy, inverse stochastic dominance, economic distance, income distribution, child care, pre-school.

JEL Codes: D63, J62, C14, I24.

\footnotetext{
${ }^{*}$ We are grateful to Rolf Aaberge, Marc Fleurbaey, Alessio Fusco, Magne Mogstad, Matias Nuñez, Erwin Ooghe, Eugenio Peluso, Flaviana Palmisano, Giuseppe Pignataro, Alain Trannoy, Dirk Van de gaer, Felix Weinhardt, Claudio Zoli for comments and discussion. This research received financial support from the French National Research Agency (TRANSINEQ grant ANR-08-JCJC-0098-01) and the Fonds National de la Recherche Luxembourg (AFR grant 5932132). Havnes also received support from The Norwegian Research Council (NRC grant 212305) and is hosted by the ESOP centre funded under NRC grant no. 179552. Lefranc acknowledges the support of the project Labex MME-DII (ANR11-LBX-0023-01).

${ }^{\dagger}$ A supplemental online appendix to this paper is available at $<$ include link $>$.

${ }^{\ddagger}$ Corresponding author: LISER, 11 Porte des Sciences, L-4366 Esch-sur-Alzette, Luxembourg. E-mails: francesco.andreoli@liser.lu (F. Andreoli), tarjei.havnes@econ.uio.no (T.Havnes) and arnaud.lefranc@u-cergy.fr (A. Lefranc).
} 


\section{Introduction}

An important goal for public policy is to promote equality of opportunity, to let individual success be determined by merit rather than by social background. Assessing whether public intervention succeeds at leveling the playing field among citizens thus represents a key issue for policy evaluation. But what criterion should be used to conduct such an evaluation? Unfortunately, while an abundant literature has been devoted to define equality of opportunity, it offers little guidance for assessing how far a given distribution is from the equality of opportunity goal. The contribution of this paper is to define a theoretical criterion of equalization of opportunity, understood as a reduction in the extent of inequality of opportunity, and to apply this criterion to policy evaluation.

Theories of equality of opportunity (EOP) draw a distinction between fair inequality, arising from differences in individual effort, and unfair inequality arising from differences in individual circumstances, i.e. the determinants of success for which society deems the individual not to be responsible (Dworkin 1981, Roemer 1998, Fleurbaey 2008). Define a type as a given set of circumstances, and an opportunity set as the set of feasible outcomes for each type. The EOP principle requires that no type is advantaged compared to other types in the sense of having access to a more favorable opportunity set. This principle allows to assess whether a given distribution satisfies equality of opportunity. However, it does not allow to compare two societies where equality of opportunity is not satisfied. This is an important limitation in many contexts, including policy evaluation and comparisons of inequality across time and space.

To address this limitation, some authors have relied on scalar indices of inequality of opportunity 1 While consistent with the EOP principles, this approach raises concerns of robustness as it relies on two restrictive assumptions. First, it requires summarizing the advantage enjoyed by a type in a scalar measure, e.g. the mean income conditional on type 2 But these scalar measures may mask important features of the distribution of opportunity. Second, the index approach relies on specific welfare functions to aggregate differences in advantage between types. Therefore, it draws on specific a priori preference

\footnotetext{
${ }^{1}$ For an overview of this approach, see Ramos and Van de gaer (2016) and Ferreira and Peragine (2016). For examples, see Bourguignon, Ferreira and Menendez (2007), Lefranc, Pistolesi and Trannoy (2008), Checchi and Peragine (2010), Almås, Cappelen, Lind, Sørensen and Tungodden (2011), Björklund, Jäntti and Roemer (2012) and Ferreira and Gignoux (2011).

${ }^{2}$ This amounts to assume that individuals are risk neutral, with respect to within-type uncertainty. Lefranc et al. (2008) assume risk aversion but rely on specific preferences.
} 
orderings that may violate the preferences of individuals in society. As a result, inequality of opportunity indices often lack robustness and generality.

Our main contribution is to alleviate these shortcomings and to develop a robust criterion that allows comparing different societies according to their degree of inequality of opportunity. We characterize a society by the opportunity sets it offers to each type. Endowed with her own preferences, each individual in society is able to compare the opportunity sets of the different types. Our equalization of opportunity (EZOP) criterion states that "Inequality of opportunity is higher in social state 0 than in social state 1", if and only if all individuals in society, regardless of their preferences, agree that the unfair advantage enjoyed by the "privileged" types is lower in state 1 than in state 0 , where different states might correspond to different countries, time periods or policy regimes.

Contrary to the index approach, our criterion does not rely on a priori value functions to assess the advantage enjoyed by each type. Instead, we use the potential preferences over opportunity sets of individuals in society and allow for heterogeneity in these preferences. This raises an important issue of identification. In practice, we only observe (at best) the opportunity sets of each type but not individual preferences. Hence it is not feasible to verify for each particular preference whether the advantage of privileged types is lower in one particular state. Instead, we would like to define a tractable condition, involving the distribution of opportunity sets alone, that would imply that our equalization criterion is satisfied. We show that such a condition can be defined using the tools of stochastic dominance. Of course, this can be achieved only within a specified family of preferences. In this paper, we focus mainly on the rank-dependent representation of preferences (Yaari 1987), although the analysis can be adapted to other classes of preferences.

The robustness and generality of our ranking criterion rests on the requirement of a consensus across individuals in their comparison of social states. We investigate the existence of such a consensus and show that our identification condition can be applied only when individuals agree on the ranking of types in each social state, i.e. when individuals agree on which types are advantaged. If individuals disagree, they cannot unanimously agree on equalization of opportunity. It is possible, however, to identify subclasses of preferences within which individuals agree on the ranking of types in each state, and to single out necessary and sufficient conditions for equalization within these subclasses of 
preferences $3^{3}$ Our criterion is demanding in requiring that equalization occurs for each pairwise comparison of a possibly large number of types. We discuss how it can be relaxed by allowing the advantage of each type to be aggregated within society. We also discuss the consequences of imperfectly observing the relevant determinants of outcome for the implementation of our equalization criterion.

Finally, we show the usefulness of our framework by applying it to the evaluation of child care policy in Norway. In this respect, we also contribute to the literature on early childhood investments.4 We follow Havnes and Mogstad $(2011,2015)$ in considering how the introduction of universally available child care in Norway affected children's adult earnings. To estimate counterfactual distributions, we estimate quantile treatment effects, exploiting the spatial and temporal variation of the expansion in a difference-in-differences setup. We allow impacts across the distribution to vary flexibly with family background. Overall, our results suggest that the child care expansion significantly equalized opportunities between children from most, though not all, family backgrounds.

The rest of the paper is organized as follows. Section 2 discusses equalization of opportunity in a simplified setting with two types. Next, section 3 considers the general case with multiple levels of effort and circumstances. Finally, section 4 presents the application.

\section{Equalization of opportunity: a simplified setting}

In this section, we define equality of opportunity and provide a formal statement of our equalization criterion in a simplified setting. Next, we discuss identification conditions.

\subsection{Definition of equality of opportunity}

Let $y \in \mathbb{R}_{+}$denote an individual outcome, and let the determinants of the outcome be partitioned into four groups: Circumstances capture determinants that are not considered legitimate sources of inequality, and are denoted by $c$. Effort captures determinants that are considered legitimate sources of inequality, and is denoted by e. Luck captures factors that are considered legitimate sources of inequality as long as they affect individual

\footnotetext{
${ }^{3}$ Aaberge, Havnes and Mogstad (2014) propose a robust welfarist criterion for ranking income distributions, based on unanimous agreement between subclasses of evaluation functions admitting the rankdependent representation.

${ }^{4}$ For surveys, see Almond and Currie (2011), Ruhm and Waldfogel (2012), or Baker (2011).
} 
outcomes in a neutral way given circumstances and effort, and is denoted by $l: 5$ Finally, outcomes are contingent on a binary social state, denoted $\pi$. All individuals in a society share the social state, but may be affected differently. For instance, $\pi=0$ may denote society without a specific policy intervention, while $\pi=1$ denotes society with the policy, or $\pi$ may indicate different periods or countries that one would like to compare.

Let a type denote the set of individuals sharing similar circumstances. Given their type, level of effort and the social state, the opportunity set offered to individuals can be summarized by the cumulative distribution function $F_{\pi}(y \mid c, e)$, or equivalently by its conditional quantile function $F_{\pi}^{-1}(p \mid c, e)$, for all ranks $p$ in $\left.[0,1]\right]^{6}$

EOP theories emphasize that inequality due to differences in circumstances is morally or politically objectionable, while inequality originating from differences in effort is legitimate. Based on this principle, equality of opportunity requires that the opportunity sets of individuals with similar effort be identical regardless of circumstances. Hence, for a given social state $\pi$, EOP requires that, for any effort $e$, for any pair of circumstances $\left(c, c^{\prime}\right)$, and for every $y$, we have:

$$
F_{\pi}(y \mid c, e)=F_{\pi}\left(y \mid c^{\prime}, e\right) .
$$

This condition embodies the core of the equality of opportunity principle, as discussed for instance in Roemer (1998), Lefranc et al. (2009) and Roemer and Trannoy (2014).7

\subsection{A criterion for equalization of opportunity}

Ranking social states The previous definition can be used to rank social states. The empirical analysis in Lefranc et al. (2009) builds on this idea. However, it distinguishes only between states where EOP is satisfied and states where EOP is not satisfied, which leads to a very partial ranking.

In order to obtain a less partial ranking, various authors have resorted to specific inequality indices in order to quantify the degree of inequality of opportunity in a given social state ${ }^{8}$ These inequality measures embody specific social preferences with respect

\footnotetext{
${ }^{5}$ For a discussion of the ethical basis that serves to substantiate each of these three classes of determinants see Lefranc, Pistolesi and Trannoy (2009) and Lefranc and Trannoy (2017).

${ }^{6}$ If the cumulative distribution function is only left continuous, we define $F_{\pi}^{-1}$ by the left continuous inverse distribution of $F_{\pi}: F_{\pi}^{-1}(p \mid c, e)=\inf \left\{y \in \mathbb{R}_{+}: F_{\pi}(y \mid c, e) \geq p\right\}$, with $p \in[0,1]$.

${ }^{7}$ This condition embodies the compensation principle advocated in Roemer (1998). It takes a neutral stance with respect to inequalities stemming from fair sources of outcome and does not resort to an additional reward principle that would further restrain the definition of equality of opportunity (Fleurbaey 2008).

${ }^{8}$ See Ramos and Van de gaer (2016) and Ferreira and Peragine (2016) for a survey.
} 
to inequality between types and to the within-type dispersion of outcomes. Inequality of opportunity indices have two main limitations. First, they lack generality, as each index relies on specific parametric formulations of social preferences. Second, they embed specific preferences of the social planner that agree with the EOP principles but might violate individual preferences over outcomes.

Our objective is to provide a robust criterion that allows comparing social states in situations where EOP is not satisfied. The intuition behind our ranking criterion is the following. If EOP is not satisfied, then individuals are not indifferent between the opportunity sets offered to different types. Behind a thin veil of ignorance, where individuals know their effort and have preferences over opportunity sets, everyone should be able to rank circumstances according to the economic advantage or disadvantage that they confer. Our criterion for ranking social states is based on the evaluation of the extent of the economic advantage enjoyed by the advantaged types in society. To ensure robustness, our equalization of opportunity criterion (EZOP) requires unanimity across all admissible preferences in society, in assessing that the unfair advantage attached to more favorable circumstances decreases.

For expositional purposes, we start by formalizing the equalization criterion in a simplified setting with only two types, $c$ and $c^{\prime}$, who exert a common effort level $e$. To simplify notation, we let $F_{\pi}($.$\left.) (resp. F_{\pi}^{\prime}().\right)$ denote the cdf of $y$ for type $c\left(\right.$ resp. $c^{\prime}$ ) at effort $e$ in policy state $\pi$, i.e. $F_{\pi}(. \mid c, e)$ (resp. $F_{\pi}\left(. \mid c^{\prime}, e\right)$ ). Section 3 provides a generalization with many types and effort levels.

The EZOP criterion We assume that each individual is endowed with cardinal preferences over risky outcomes. Let $W(F)$ denote the utility of a lottery with cumulative distribution $F$, and let $\mathcal{P}$ denote the class of individual preferences. For an individual with preferences $W \in \mathcal{P}$, the economic advantage or disadvantage of type $c$ relative to type $c^{\prime}$ in social state $\pi$ is denoted $\Delta_{W}\left(F_{\pi}, F_{\pi}^{\prime}\right) \equiv W\left(F_{\pi}\right)-W\left(F_{\pi}^{\prime}\right)$. This quantity is positive if the individual with preferences $W$ prefers $F_{\pi}$ to $F_{\pi}^{\prime}$, while it is equal to zero if EOP holds between types $c$ and $c^{\prime}$. We refer to the absolute value of the welfare gap as the economic distance between types according to preferences $W$ 9

The equalization of opportunity criterion rests on the difference in economic advantage

\footnotetext{
${ }^{9}$ For a discussion, see Chakravarty and Dutta (1987).
} 
across social states, as captured by the following definition:

Definition 1 (EZOP: equalization of opportunity between two types) Moving from state $\pi=0$ to $\pi=1$ equalizes opportunity between circumstances $c$ and $c^{\prime}$, at effort $e$ on the set of preferences $\mathcal{P}$, if and only if, for all preferences $W \in \mathcal{P}$, we have: $\left|\Delta_{W}\left(F_{0}, F_{0}^{\prime}\right)\right| \geq\left|\Delta_{W}\left(F_{1}, F_{1}^{\prime}\right)\right|$.

The equalization of opportunity criterion defines a social ordering requiring unanimity among potential individual preferences. It has several key properties that are worth emphasizing. First, in line with the theory of EOP, ranking state 1 above state 0 requires that the unfair economic advantage enjoyed by the privileged type be smaller in state 1 than in 0 . Second, the criterion satisfies an anonymity condition with respect of the identity of the advantaged type: Only the absolute value of the economic advantage, but not its sign, should matter for assessing equalization of opportunity. Third, it requires that the ranking be robust to a broad class of individual preferences. Fourth, in line with most of the inequality literature, the EZOP criterion focuses only on the difference in welfare across types and not on the level of welfare in each social state. This view implies that an overall reduction in aggregate welfare in society could lead to a reduction in inequality of opportunity, provided that the welfare gaps across types also falls. To address such cases, the EZOP criterion can be complemented by further requiring that average welfare or the welfare of the worse-off type does not fall when moving from state 0 to state 110 Last, our EZOP criterion takes into account the absolute welfare gap between types. As a complement to this absolute perspective, a relative view can be developed by focusing on the distribution of income shares across types, as discussed in section 3.2 below.

\subsection{Identification under the rank-dependent utility model}

The identification problem The EZOP criterion is contingent on the choice of the class of preferences $\mathcal{P}$. If the set of individual preferences $W$ in society was known, we could directly check whether the equalization condition holds. In practice, we know only the outcome distributions under the two policy states but not individual preferences. Therefore, the condition in definition 1 cannot be directly assessed.

To make the equalization criterion relevant, we need to reformulate it in terms of

\footnotetext{
${ }^{10}$ Peragine (2002) offers an alternative criterion that focuses on social welfare improvement, in a sequential way, by giving priority to welfare gains for the least privileged types.
} 
a restriction that involves only the outcome distributions of the different types in the alternative states. This cannot be achieved without imposing restrictions on the class $\mathcal{P}$ of individual preferences. Two possible alternative representations of preferences under risk have been widely studied and adopted in decision theory: The expected utility model and the rank-dependent model of Yaari (1987). In the rest of the paper, we focus on the rank-dependent class, which we denote by $\mathcal{R} 11$ In the rest of this section we concentrate on the following question: What minimal conditions need to be imposed on the set of distributions $F_{0}, F_{0}^{\prime}, F_{1}, F_{1}^{\prime}$ to ensure that equalization is satisfied for all preferences in $\mathcal{R}$ ?

The rank-dependent model assumes that the welfare derived from a risky distribution $F$ can be written as a weighted average of all possible realizations, where the weights are a function of the rank of the realization in the distribution of outcomes. Formally, let $w(p) \geq 0$ denote the weight assigned to the outcome at percentile $p$. The welfare derived from $F$ can then be written as 12

$$
W(F)=\int_{\mathbb{R}_{+}} w(F(y)) y d y=\int_{0}^{1} w(p) F^{-1}(p) d p .
$$

Under the rank-dependent model, the economic distance between types is given by:

$$
\left|\Delta_{W}\left(F, F^{\prime}\right)\right|=\left|\int_{0}^{1} w(p)\left(F^{-1}(p)-F^{\prime-1}(p)\right) d p\right|=\left|\int_{0}^{1} w(p) \Gamma\left(F, F^{\prime}, p\right) d p\right|,
$$

where $\Gamma\left(F, F^{\prime}, p\right)$ is the cumulative distribution gap between $F$ and $F^{\prime}$. We refer to the graph of $\Gamma(., .,$.$) as the gap curve and to the graph of |\Gamma(., .,)$.$| as the absolute gap curve.$

Necessary condition for EZOP From equation [2], a necessary condition for EZOP is that the cumulative distribution gap under $\pi=1$ be smaller, in absolute value, compared to the gap under $\pi=0$, at all percentiles. We refer to this as absolute gap curve dominance of $\pi=1$ over $\pi=0$.

Proposition 1 EZOP is satisfied on the set of preferences $\mathcal{R} \Rightarrow \forall p \in[0,1],\left|\Gamma\left(F_{1}, F_{1}^{\prime}, p\right)\right| \leq$

\footnotetext{
${ }^{11}$ In addition to their tractability in empirical evaluations, the rank-dependent family of preferences resolves important paradoxes in the theory of choice under risk (see e.g. Allais 1953, Kahneman and Tversky 1979, Quiggin 1981). It also has a unique position in empirical welfare analysis in providing theoretical underpinnings for the widely used Gini index (see e.g. Sen 1974). Our framework is not confined, however, to the rank-dependent family, and could be extended to other families of preferences. For instance, equalization conditions can be derived for the class of Von Neumann expected utility preferences. The online appendix provides such conditions under first order dominance.

${ }^{12}$ Formally, one requires that $w(p) \geq 0 \forall p \in[0,1]$ and $\widetilde{w}(p)=\int_{0}^{p} w(t) d t \in[0,1]$ is such that $\widetilde{w}(1)=1$.
} 
$\left|\Gamma\left(F_{0}, F_{0}^{\prime}, p\right)\right|$

N.B. Proofs of this and subsequent propositions are given in the online supplemental appendix, section A.

The intuition of the proof is that if the absolute gap curve increases, there always exists a preference in $\mathcal{R}$ for which the unfair economic advantage increases. Note that absolute gap curve dominance is not a sufficient condition for EZOP. Whether a reduction in the gap between type $c$ and $c^{\prime}$ amounts to a reduction in advantage, will depend on which of the two groups is considered to be advantaged. Because the assessment of which type is advantaged may differ over the set of possible preferences, the requirement for EZOP over all possible preferences must be stronger than what is imposed by absolute gap curve dominance. For instance, assume that the distribution of type $c$ dominates the distribution of type $c^{\prime}$ over some interval. This does not imply, in the general case, that type $c$ dominates $c^{\prime}$ over the entire support of the distribution. Henceforth, some preferences might rank $c^{\prime}$ better than $c$. Now assume that gap curve dominance is satisfied over this interval and that gap curves are similar in both social states otherwise. In this case, preferences that rank $c^{\prime}$ better than $c$ will conclude that the cardinal advantage of $c^{\prime}$ has increased. This contradicts EZOP.

Necessary and sufficient condition under stochastic dominance A corollary of the previous discussion is that if individuals agree on the ranking of types, then they should also agree in their ranking of social states under gap curve dominance. We now examine this specific case.

As discussed in Muliere and Scarsini (1989), among others, unanimity in ranking distributions $F_{\pi}$ better than $F_{\pi}^{\prime}$ will be achieved for all preferences in $\mathcal{R}$ if and only if distribution $F_{\pi}$ stochastically dominates distribution $F_{\pi}^{\prime}$. This is equivalent to requiring inverse stochastic dominance at order one, which we denote $F_{\pi} \succ_{I S D 1} F_{\pi}^{\prime}$. This holds whenever the graph of $F_{\pi}^{-1}$ lies above the graph of $F_{\pi}^{\prime-1}{ }^{13}$

In this section, we assume that this condition is satisfied 14 If so, all preferences in $\mathcal{R}$

\footnotetext{
${ }^{13}$ Note that stochastic dominance and inverse stochastic dominance are equivalent at the first and second order. The difference is that the dominance condition in the latter case is expressed in the space of realizations (through the quantile function) while in the former case it is expressed in the space of probabilities (through the cdf).

${ }^{14}$ Since $c$ and $c^{\prime}$ play a symmetric role in the definition of EZOP, which type dominates the other is irrelevant. Hence we make the neutral assumption that the distribution of type $c$ dominates the distribution of type $c^{\prime}$, under both policy regimes.
} 
unanimously rank type $c$ better than type $c^{\prime}$. A fall in the cumulative distribution gap then has unambiguous consequences for the change in the economic distance between types. In fact, since the sign of the cumulative distribution gap is constant across all percentiles, the economic distance can be expressed as an increasing function of the absolute income gap: $\left|\Delta_{W}\left(F, F^{\prime}\right)\right|=\int_{0}^{1} w(p)\left|\Gamma\left(F, F^{\prime}, p\right)\right| d p$. This leads to the following proposition:

Proposition 2 If $\forall \pi F_{\pi} \succ_{I S D 1} F_{\pi}^{\prime}$ then: EZOP over the set of preferences $\mathcal{R} \Leftrightarrow \forall p \in$ $[0,1], \Gamma\left(F_{0}, F_{0}^{\prime}, p\right) \geq \Gamma\left(F_{1}, F_{1}^{\prime}, p\right)$.

This proposition establishes that when individuals agree on the ranking of types, gap curve dominance provides a necessary and sufficient condition for EZOP. This contrasts with the situation where preferences do not agree on the ranking of types, in which case gap curve dominance provides only a necessary condition for EZOP. In order to evaluate EZOP in such situations, we next consider refinements on the admissible set of preferences.

Restricted consensus on EZOP When types cannot be ranked unambiguously, the cumulative distribution gap is no longer sufficient to infer EZOP. Our objective is to identify the minimal refinement on the set of admissible preferences that allows unambiguous assessments of equalization of opportunity. In line with Aaberge et al. (2014), we show that it is always possible to find a subset of $\mathcal{R}$ over which individuals agree on the ranking of types. Furthermore, on this subset, one can establish a necessary and sufficient condition for equalization of opportunity.

Let us first consider the special case where $F_{\pi}$ second order stochastic dominates $F_{\pi}^{\prime}$ for all $\pi \in\{0,1\}$, which we denote $F_{\pi} \succ_{I S D 2} F_{\pi}^{\prime}$. This holds whenever the graph of the integral of $F_{\pi}^{-1}$ with respect to $p$ (the Generalized Lorenz curve) lies above the graph of the corresponding integral of $F_{\pi}^{\prime-1}$. Define $\mathcal{R}^{2} \subset \mathcal{R}$ as the set of risk-averse rankdependent preferences ${ }^{15}$ As is well known, all risk averse preferences rank distribution functions consistently with second order dominance. It follows that all preferences in $\mathcal{R}^{2}$ will rank type $c$ better than $c^{\prime}$ in both states. Furthermore, the advantage of $c$ over $c^{\prime}$ can be expressed as an increasing function of the integral of the cumulative distribution gap. Analogous to the above, a necessary and sufficient condition for EZOP over the set of preferences $\mathcal{R}^{2}$ is then that the integrated cumulative distribution gap falls at all percentiles. This is established in the following proposition:

\footnotetext{
${ }^{15}$ This set contains all evaluation functions with weights decreasing in outcomes, i.e. that have $w^{\prime}(p)<0$.
} 
Proposition 3 If $\forall \pi F_{\pi} \succ_{I S D 2} F_{\pi}^{\prime}$ then: EZOP over the set of preferences $\mathcal{R}^{2} \Leftrightarrow \forall p \in$ $[0,1], \int_{0}^{p} \Gamma\left(F_{0}, F_{0}^{\prime}, t\right) d t \geq \int_{0}^{p} \Gamma\left(F_{1}, F_{1}^{\prime}, t\right) d t$

Finally, consider the case where distributions cannot be ranked by second order dominance. In this case, consensus over the ranking of types cannot be reached in the class $\mathcal{R}^{2}$. However, it is possible to refine the set of preferences to where they agree on the ranking of types. Following Aaberge (2009), consider the subset of preferences $\mathcal{R}^{k}$ defined by:

$$
\mathcal{R}^{k}=\left\{W \in \mathcal{R} \mid(-1)^{i-1} \cdot \frac{d^{i} \widetilde{w}(p)}{d p^{i}} \geq 0, \quad \frac{d^{i} \widetilde{w}(1)}{d p^{i}}=0 \forall p \in[0,1] \text { and } i=1, \ldots, k\right\}
$$

where $\widetilde{w}(p)=\int_{0}^{p} w(t) d t$ is the cumulative weighting scheme. The sequence of subsets of the type $\mathcal{R}^{k}$ defines a nested partition of $\mathcal{R}$ where $\mathcal{R}^{k} \subset \mathcal{R}^{k-1} \subset \ldots \subset \mathcal{R} 16$

Various papers have examined the relationship between inverse stochastic dominance and the ordering of distributions according to preferences in $\mathcal{R}^{k}$ (Muliere and Scarsini 1989, Zoli 2002). Aaberge et al. (2014) provide a general treatment and show that for any order $k$ all preferences in $\mathcal{R}^{k}$ will prefer $F_{\pi}$ over $F_{\pi}^{\prime}$ if and only if $F_{\pi}$ inverse stochastic dominates $F_{\pi}^{\prime}$ at order $k$. Furthermore, as we show in the online appendix, any pair of distributions can always be ranked by inverse stochastic dominance, for a sufficiently high finite order. Define $\kappa$ as the minimal order at which $F_{\pi}$ and $F_{\pi}^{\prime}$ can be ranked using inverse stochastic dominance in both states, and denote $k^{\text {th }}$ order inverse stochastic dominance by $\succ_{I S D k}$. Without loss of generality, assume that $F_{\pi} \succ_{I S D \kappa} F_{\pi}^{\prime}$ for all $\pi \in\{0,1\}$, such that preferences in $\mathcal{R}^{\kappa}$ agree on the ranking of types in both states ${ }^{17}$

To proceed, it is helpful to introduce the following notation:

$$
\Lambda_{\pi}^{2}(p)=\int_{0}^{p} F_{\pi}^{-1}(t) d t \quad \text { and } \quad \Lambda_{\pi}^{k}(p)=\int_{0}^{p} \Lambda_{\pi}^{k-1}(t) d t, \text { for } k=3,4, \ldots
$$

For notational simplicity, we let $\Lambda_{\pi}^{\prime k}$ denote $\Lambda_{\pi}^{k}$ evaluated over the distribution $F_{\pi}^{\prime}$ rather than $F_{\pi}$. With these notations, inverse stochastic dominance of order $k$ is defined as $\Lambda_{\pi}^{k}(p)>\Lambda_{\pi}^{\prime k}(p)$ for all $p \in[0,1]$. In line with the notation above, also define $\Gamma^{k}\left(F_{\pi}, F_{\pi}^{\prime}, p\right)=$ $\Lambda_{\pi}^{k}(p)-\Lambda_{\pi}^{\prime k}(p)$ as the cumulative distribution gap integrated at order $k-1$.

If, for all $\pi \in\{0,1\}, F_{\pi} \succ_{I S D \kappa} F_{\pi}^{\prime}$, then for all preferences $W \in \mathcal{R}^{\kappa}$ the advantage

\footnotetext{
${ }^{16}$ Note that $k$ is a measure of the effect of a precise sequence of restrictions on all possible cumulative weighting schemes $\widetilde{w}(p)$ defined on $\mathcal{R}$. Hence, $k$ indicates the risk attitude of preferences contained in $\mathcal{R}^{k}$.

${ }^{17} \mathrm{~A}$ larger $\kappa$ reduces preference heterogeneity in $\mathcal{R}^{\kappa}$, making ranking agreement more likely, yet less robust. Also note that order $k$ inverse stochastic dominance implies order $k+1$ dominance.
} 
of type $c$ over type $c^{\prime}$ under policy $\pi$ is an increasing function of $\Gamma^{\kappa}\left(F_{\pi}, F_{\pi}^{\prime}, p\right)$. As a consequence, EZOP will be satisfied on the set of preferences $\mathcal{R}^{\kappa}$ if and only if $\Gamma^{\kappa}\left(F_{\pi}, F_{\pi}^{\prime}, p\right)$ is smaller under $\pi=1$ than under $\pi=0$. This is established in the following proposition:

Proposition 4 If $\forall \pi F_{\pi} \succ_{I S D \kappa} F_{\pi}^{\prime}$ then: EZOP over the set of preferences $\mathcal{R}^{\kappa} \Leftrightarrow \forall p \in$ $[0,1], \Gamma^{\kappa}\left(F_{0}, F_{0}^{\prime}, p\right) \geq \Gamma^{\kappa}\left(F_{1}, F_{1}^{\prime}, p\right)$.

Proposition 4 establishes a necessary and sufficient condition for EZOP under a less stringent dominance condition than in propositions 2 and 3 . At the same time, the set of preferences over which it allows to identify EZOP is more restrictive. Finally, since there always exists an integer $\kappa$ that allows ranking types, proposition 4 establishes a necessary condition for EZOP over the entire class $\mathcal{R}$.

\subsection{Discussion}

Several features of our equalization criterion are worth discussing further. First, our criterion relies on the individuals' own preferences, rather than on an external social welfare function. This is consistent with the no-envy criterion (Fleurbaey 2008), which requires that individuals with given preferences and effort be indifferent between the opportunity sets of the different types. Hence, the advantage enjoyed by privileged types represents a measure of the degree of envy, for given preferences. Second, the criterion is general, in the sense that it does not place any restriction on the preferences of individuals. The degree of heterogeneity of preferences across the population is clearly unobservable. The focus is therefore on the class of potential preferences these individuals may have. Third, the criterion does not in itself require that individuals agree on the ranking of types, only that they agree on the reduction in the absolute gap between the different types. In other words, our criterion requires a consensus on the reduction of the advantage but not on the identity of the advantaged type. Finally, the criterion does not require summarizing the opportunity sets of the different types by a scalar measure as is often done in the literature on the measurement of inequality of opportunity.

The results obtained under the rank-dependent assumption also call for further comments. They lead us to distinguish between two cases. The case where individuals agree on the ranking of types under each social state is straightforward, as proposition 2 provides a necessary and sufficient condition for equalization of opportunity. In the case where indi- 
viduals do not agree on the ranking of types, however, proposition 1 provides a necessary condition for equalization. Violation of this condition rules out equalization. Otherwise, proposition 4 allows one to endogenously identify a restricted set of preferences over which unanimity might be reached regarding equalization of opportunity. Of course, this only provides a partial judgment over equalization of opportunity. In fact, the higher the order of $\kappa$ required to successfully rank opportunity sets, the less general the judgment will be.

The restrictions on preferences required to achieve a consensus on the ranking of types may however be more directly informative. When weak restrictions are required to achieve a consistent ranking, then most individuals should agree on which type is advantaged. On the contrary, when stronger restrictions are required, there may be widespread disagreement on which type is advantaged. In this case, one might argue that a weak form of equality of opportunity already prevails. Lefranc et al. (2009) introduce the notion of weak equality of opportunity to single out situations where the opportunity sets differ across types but cannot be ranked unanimously among agents with risk-averse preferences. By capturing the degree of consensus about the advantaged type among potential preferences, $\kappa$ helps generalize this notion of weak equality of opportunity.

To summarize, when there may be widespread disagreement on which type is advantaged (high $\kappa$ ), our criterion provides a very partial condition for consensus on equalization of opportunity, although this admittedly corresponds to a case of weak inequality of opportunity. On the contrary, when there is large agreement on which type is advantaged (low $\kappa$ ), our equalization condition becomes least partial and turns into a necessary and sufficient condition for EZOP in the case where there is full consensus on the identity of the advantaged type $(\kappa=1)$.

\section{Equalization of opportunity: generalization}

In the general case, opportunity equalization has to be assessed with more than two circumstances, across many effort levels. When effort is observable, one possibility is to extend the EZOP comparisons to all pairs of circumstances at every effort level, or to study meaningful aggregations of these judgements. We discuss both extensions in this section. Identification criteria when effort is not observable are also discussed, in order to provide relevant notions of equalization that can still be used in applied analysis, under 
observability constraints.

\subsection{Extending the EZOP criterion to multiple circumstances}

We consider the case in which there are $T$ types. Let $C=\left\{c_{1}, \ldots, c_{i}, \ldots c_{T}\right\}$ denote the set of possible circumstances. For simplicity, we assume a single effort level $e$. The results of this section can be easily extended to multiple effort levels (see section 3.3).

A straightforward extension of definition 1 to multiple circumstances is to require that for every possible pair of circumstances, the unfair gap falls when moving from social state $\pi=0$ to $\pi=1$. This is given by the following definition.

Definition 2 (EZOP between multiple types) Moving from state $\pi=0$ to $\pi=1$ equalizes opportunity over the set of circumstances $C$, at effort $e$, on the set of preferences $\mathcal{P}$, if and only if, for all preferences $W \in \mathcal{P}$, for all $(i, j) \in\{1, \ldots, T\}^{2}$, we have: $\left|\Delta_{W}\left(F_{0}\left(. \mid c_{i}, e\right), F_{0}\left(. \mid c_{j}, e\right)\right)\right| \geq\left|\Delta_{W}\left(F_{1}\left(. \mid c_{i}, e\right), F_{1}\left(. \mid c_{j}, e\right)\right)\right|$.

Again, this generalized form of EZOP cannot be verified in practice without specifying the class of preferences. In the class $\mathcal{R}$, the results of propositions 2 and 4 generalize easily to the multivariate case. For every pair $(i, j)$, let $\kappa_{i j}$ denote the minimal order at which $F_{\pi}\left(. \mid c_{i}, e\right)$ and $F_{\pi}\left(. \mid c_{j}, e\right)$ can be ranked according to inverse stochastic dominance, for all $\pi$. According to proposition 4, integrated gap curve dominance for each pair of types $c_{i}$ and $c_{j}$ provides a necessary and sufficient condition for EZOP between the two types over the subclass $\mathcal{R}^{\kappa_{i j}}$. This condition is, however, only necessary when looking at the whole class $\mathcal{R}$.

Proposition 5 EZOP between multiple types over the set of preferences $\mathcal{R} \Rightarrow \forall(i, j) \in$ $\{1, \ldots, T\}, \forall p \in[0,1],\left|\Gamma^{\kappa_{i j}}\left(F_{0}\left(. \mid c_{i}, e\right), F_{0}\left(. \mid c_{j}, e\right), p\right)\right| \geq\left|\Gamma^{\kappa_{i j}}\left(F_{1}\left(. \mid c_{i}, e\right), F_{1}\left(. \mid c_{j}, e\right), p\right)\right|$.

The proof is based on the same arguments used in the proof of proposition 4. Differently from this proposition, while we know that $F_{\pi}\left(. \mid c_{i}, e\right)$ and $F_{\pi}\left(. \mid c_{j}, e\right)$ can be ranked at the order of dominance $\kappa_{i j}$, the direction of dominance is a priori undetermined. This explains why gap dominance should hold in absolute value.

Definition 2 makes the "identity" of each type relevant for defining equalization of opportunity, since the extent of advantage between any pair of types $\left(c_{i}, c_{j}\right)$ under $\pi=0$ is compared with the extent of advantage between the same two types under $\pi=1$. One may 
challenge this view and claim that only the magnitude of the gaps (and not the identity of the types involved) is relevant for defining equalization of opportunity. One way to implement this idea is through anonymous criteria of equalization between multiple types, where the type label is replaced with the type rank in the order of advantage. We formally develop these criteria and provide testable implications in the online appendix.

\subsection{Aggregation across circumstances}

Definition 2 is demanding and may fail to be satisfied empirically, as it requires that the welfare gap falls for every pair from a possibly large number of types. As a result, the EZOP criterion allows only a partial ordering of social states. Furthermore, it might be argued that a small increase in the opportunity gap between two types might be compensated by a fall in the opportunity gap between another pair of types. This suggests aggregating welfare gaps across pairs of circumstances into a scalar measure.

Such aggregation requires selecting two value functions. The first function, $W$, evaluates the opportunities available to each type. The second function, $V$, aggregates the welfare levels across types into a single value of social welfare. For a pair of functions $V$ and $W$, one can define an Inequality of Opportunity indicator $\left(I O_{W}^{V}\right)$ for each social state $\pi$ :

$$
I O_{W}^{V}(\pi)=V\left(W\left(F_{\pi}\left(. \mid c_{1}, e\right)\right), \ldots, W\left(F_{\pi}\left(. \mid c_{T}, e\right)\right)\right)
$$

Restrictions have to be imposed on $V$ in order to obtain a scalar measure that is consistent with the EZOP principle defined in the previous section. Note that, for a specific function $W$, the inequality condition that appears in definition 2 amounts to requesting that the vector of type-specific welfare levels in state $\pi=1,\left(W\left(F_{1}\left(. \mid c_{1}, e\right)\right), \ldots, W\left(F_{1}\left(. \mid c_{T}, e\right)\right)\right)$ can be obtained from the same vector in state $\pi=0$, by applying a series of progressive Pigou-Dalton welfare transfers and, possibly, a lump-sum welfare transfer to all types. This implies that the function $V$ should be consistent with the Pigou-Dalton transfers principle and translation invariant. Hence, up to an increasing transformation, the function $V$ should be an absolute inequality index (Moyes 1987).

Our Inequality of Opportunity indicator is thus a measure of between-types welfare inequality, computed on the basis of a specific function $W$, using an absolute inequality index. If EZOP is satisfied for a particular $W$, then $I O_{W}^{I A}(1) \leq I O_{W}^{I A}(0)$ for any absolute 
inequality index, denoted $I A$.

As an example, consider the function $V$ associated with the absolute Gini coefficient (Chakravarty 1988), which is simply the standard Gini coefficient multiplied by the mean. When types have different relative frequencies in the population, $p_{c}$, a natural extension is to account for type frequency when computing between-type inequality. This yields the following inequality of opportunity indicator:

$$
I O_{W}^{G i n i}(\pi)=\sum_{i=1}^{T-1} \sum_{j=i+1}^{T} p_{c_{i}} p_{c_{j}}\left|W\left(F_{\pi}\left(. \mid c_{i}, e\right)\right)-W\left(F_{\pi}\left(. \mid c_{j}, e\right)\right)\right| .
$$

$I O_{W}^{\text {Gini }}$ equals the average absolute welfare gap, across all pairs of circumstances, computed for function $W$.

Equations (3) and (4) encompass several inequality of opportunity indices suggested in the literature. Lefranc et al. (2008) introduce the Gini Opportunity index defined as:

$$
G O(\pi)=\frac{1}{\mu} \sum_{i=1}^{T-1} \sum_{j=i+1}^{T} p_{c_{i}} p_{c_{j}}\left|\mu_{c_{i}}\left(1-G_{c_{i}}\right)-\mu_{c_{j}}\left(1-G_{c_{j}}\right)\right| .
$$

The index is obtained from $I O_{W}^{G i n i}$ by plugging in the function $W\left(F_{\pi}(. \mid c, e)\right)=\frac{\mu_{c}}{\mu}\left(1-G_{c}\right)$, where $\mu_{c} / \mu$ is the ratio between the mean outcome conditional on circumstance $c$ and the population mean, while $G_{c}$ is the Gini coefficient for type $c$. Alternatively, using the function $W\left(F_{\pi}(. \mid c, e)\right)=\frac{\mu_{c}}{\mu}$ in (4) yields the inter-type relative Gini coefficient 18

The indices introduced in Checchi and Peragine (2010) and Ferreira and Gignoux (2011) can also be seen as special cases of equation (3). Both papers suggest measuring inequality of opportunity by applying a standard inequality index $I$ to the smoothed income distribution, i.e. the income distribution where individual incomes are replaced by the typespecific mean incomes. Hence, their inequality index can be written as $I\left(\mu_{c_{1}}, \ldots, \mu_{c_{T}}\right)$. In terms of the notation in equation (3), this implies that the function $V$ is replaced by a standard inequality index. Since both papers advocate using relative inequality indices, one may worry that this produces inequality indices that are not consistent with the EZOP criterion. However, note that in the case of relative inequality indices we have: $I\left(\mu_{c_{1}}, \ldots, \mu_{c_{T}}\right)=I\left(\frac{\mu_{c_{1}}}{\mu}, \ldots, \frac{\mu_{c_{T}}}{\mu}\right)$. Thus, one can view the inequality of opportunity indices of Checchi and Peragine (2010) and Ferreira and Gignoux (2011) as relying on

\footnotetext{
${ }^{18}$ For a complete survey of Gini-type indices for equality of opportunity sets, see Weymark (2003).
} 
the relative type-specific mean income to evaluate the expected welfare, $W$, of any type. Furthermore, one should stress that this specific measure of welfare has a constant mean equal to one, in each state. Hence, for such an evaluation function $W$, requesting that the function $V$ is translation invariant is irrelevant and we can simply request that the function $V$ is consistent with the Pigou-Dalton principle, which is indeed satisfied by any relative inequality index $I$. An important implication of this discussion is that a relative approach to inequality of opportunity can be developed, within the setting of this paper, by applying the gap curve dominance criterion to the mean-normalized income distributions. This allows generalizing the relative inequality of opportunity indices introduced in the literature 19

\subsection{Aggregation in the effort dimension}

Let us now consider a situation where effort can be summarized by a scalar indicator $e \in \mathbb{R}^{+}$. We refer to the distribution of effort within a type as $G(e \mid c, \pi)$.

Assume first that effort is realized and observable. This corresponds to what has been referred to in the EOP literature as an ex post situation (Fleurbaey and Peragine 2013). A straightforward extension of definition 2 to the multiple effort setting can be made by requiring equalization to hold at every effort level, which can be assessed with ideal data.

In most existing data sets, however, information on effort is missing. In this context, it is only possible to observe for each type its outcome distribution, given by:

$$
F_{\pi}(y \mid c)=\int_{E} F_{\pi}(y \mid c, e) d G(e \mid c, \pi)
$$

In the presence of luck, the distribution of outcome of a given type arises from a mixture of luck and effort factors.

The ex-ante approach The distributions $F_{\pi}(. \mid c)$ are interesting in their own right and relevant for opportunity equalization. Each distribution captures the opportunity sets associated to different types in an ex ante perspective, i.e. before the effort choices are

\footnotetext{
${ }^{19}$ Both Checchi and Peragine (2010) and Ferreira and Gignoux (2011) introduce an alternative measure of inequality of opportunity, defined as the share of inequality of opportunity in total inequality of outcome. Note, however, that our EZOP criterion takes into account only between-types inequality and is insensitive to inequality arising from effort or luck. In the same spirit, however, our inequality of opportunity indicator could be divided by a measure of welfare inequality.
} 
made. If EZOP judgements are made without knowing in advance what individual effort choices will be, the ex post level of effort could be treated as luck. This amounts to assuming that all individuals in a type exert similar effort. One may further assume that effort levels are comparable across types, as discussed below. This comes close in spirit to the analysis of Van de gaer (1993). In this case, equalization should be decided on the basis of the outcome distributions of each type, $F_{\pi}(y \mid c)$ :

Definition 3 (Ex ante EZOP between multiple types) Moving from state $\pi=0$ to $\pi=1$ equalizes opportunity ex ante over the set of circumstances $C$ on the set of preferences $\mathcal{P}$ if and only if for all preferences $W \in \mathcal{P}$, for all $(i, j) \in\{1, \ldots, T\}^{2}$, we have: $\left|\Delta_{W}\left(F_{0}\left(. \mid c_{i}\right), F_{0}\left(. \mid c_{j}\right)\right)\right| \geq\left|\Delta_{W}\left(F_{1}\left(. \mid c_{i}\right), F_{1}\left(. \mid c_{j}\right)\right)\right|$.

According to this definition, opportunities are equalized if preferences agree that the gaps between the expected opportunity sets associated with every pair of circumstances fall with the change in social state. When $\mathcal{P}=\mathcal{R}$, proposition 5 can be used to identify ex ante EZOP 20 This suggests using empirical gap curves based on observable distributions, conditional on circumstances alone, to assess ex ante EZOP.

The relationship between ex ante and ex post EZOP Ex ante and ex post EZOP correspond to different concepts of equalization. Empirically assessing the ex ante perspective is less demanding in terms of data. Yet a key question is whether the ex ante distributions can also be used to evaluate ex post EZOP.

First consider the Roemerian setting where luck plays no role. Individuals with circumstances $c$ and effort $e$ in state $\pi$ are assigned a single value of outcome $Y_{\pi}(c, e)$. Hence, ex post equalization amounts to require that for all $\left(c, c^{\prime}\right)$ and all $e:\left|Y_{0}(c, e)-Y_{0}\left(c^{\prime}, e\right)\right| \geq$ $\left|Y_{1}(c, e)-Y_{1}\left(c^{\prime}, e\right)\right|$. The Roemerian concept of effort requires, on a priori grounds, that effort be defined such that its distribution is independent of type ${ }^{21}$ Roemer further assumes that the outcome function $Y_{\pi}(c, e)$ is strictly increasing in $e$. In this case, the individual effort within a type can be identified by the rank in the type-specific outcome distribu-

\footnotetext{
${ }^{20}$ In this case, proposition 5 has to be reformulated using distributions of outcomes conditional on circumstances. A necessary condition for ex-ante EZOP between multiple types is that $\forall(i, j) \in\{1, \ldots, T\}$, $\forall p \in[0,1],\left|\Gamma^{\kappa_{i j}}\left(F_{0}\left(. \mid c_{i}\right), F_{0}\left(. \mid c_{j}\right), p\right)\right| \geq\left|\Gamma^{\kappa_{i j}}\left(F_{1}\left(. \mid c_{i}\right), F_{1}\left(. \mid c_{j}\right), p\right)\right|$.

${ }^{21}$ The argument is that since individuals cannot be held responsible for their type, they should not be held accountable for the association between their "effort" and their type. One may push the argument further and require that the distribution of effort is also independent of the state. For a complete discussion of the conditions of identification of equality of opportunity in Roemer's model, see O'Neill, Sweetman and Van De Gaer (2000) and Lefranc et al. (2009).
} 
tion: $e \equiv p=F_{\pi}(y \mid c)$ and we have $Y_{\pi}(c, e)=F_{\pi}^{-1}(p \mid c)$. Ex post EZOP in this setting is thus equivalent to requiring ex ante absolute gap curve dominance, i.e. for all $p \in[0,1]$ : $\left|F_{0}^{-1}(p \mid c)-F_{0}^{-1}\left(p \mid c^{\prime}\right)\right| \geq\left|F_{1}^{-1}(p \mid c)-F_{1}^{-1}\left(p \mid c^{\prime}\right)\right|$. Hence, ex post EZOP can be tested with ex ante data alone. When the ex ante distributions can be ranked according to stochastic dominance, we can establish the equivalence between gap curve dominance and ex ante dominance. This implies that ex ante EZOP is equivalent to ex post EZOP when types can be ranked ex ante.

Next, let us turn to the general setting where luck and effort distributions are not degenerate. In this case, the relationship between ex ante and ex post equalization cannot be established without further assumptions. Consider first a simple example with two circumstances, $c$ and $c^{\prime}$, and many effort levels. Assume that for all effort levels, type $c$ dominates $c^{\prime}$ at the first order. In this case, ex post EZOP requires that for all $e,\left|F_{0}(y \mid c, e)-F_{0}\left(y \mid c^{\prime}, e\right)\right| \geq\left|F_{1}(y \mid c, e)-F_{1}\left(y \mid c^{\prime}, e\right)\right|$. Assume further that effort is distributed independently of type and state. Under these two assumptions, we have, using (5): $\left|F_{\pi}(y \mid c)-F_{\pi}\left(y \mid c^{\prime}\right)\right|=\int\left|F_{\pi}(y \mid c, e)-F_{\pi}\left(y \mid c^{\prime}, e\right)\right| d G(e)$. This allows to establish that rejection of ex ante EZOP implies rejection of ex post EZOP. However, this is only valid under the two maintained assumptions. Unfortunately, these assumptions cannot be tested empirically, without observing effort. This shows that in the general case, ex post equalization cannot be identified using ex ante comparisons.

\section{Child care expansion and equalization of opportunity in Norway}

Recently, policymakers both in the US and in Europe are pushing for expanding access to child care, in an effort to alleviate early life differences across socioeconomic groups. Indeed, early childhood investments are often seen as the means par excellence to equalize life chances (Blau and Currie 2006, Heckman and Masterov 2007). To illustrate the usefulness of our framework for policy evaluations, we now apply it to evaluate the long term impact of a large scale child care reform in Norway.

The Kindergarten Act passed the Norwegian parliament in June 1975. It assigned responsibility for child care to local municipalities and was followed by large increases in federal funding. The reform constituted a substantial positive shock to the supply of 
subsidized child care, which had been severely constrained by limited public funds. The child care coverage rate for 3 to 6 year olds increased from less than $10 \%$ in 1975 to over $28 \%$ by 19792

Our objective is to assess whether the expansion of child care equalized opportunity among Norwegian children. The outcome variable we focus on is individual yearly earnings at age 30-36. Our circumstance variable is parental earnings during early childhood. Havnes and Mogstad (2011) show that the child care expansion had, on average, positive long-run effects on children's education and labour market attachment. Havnes and Mogstad (2015) document that the effects were highly heterogenous: gains were clustered in the lower end of the overall earnings distribution, and were on average larger for children from disadvantaged backgrounds. Whether the distribution of gains for disadvantaged children dominates the one of advantaged children remains however an open question.

We extend on Havnes and Mogstad (2015) by looking at the full distributional consequences of the child care expansion within family background, and by bringing the EZOP framework to bear on these results. Specifically, we examine to what extent the expansion of child care equalized children's earnings distributions as adults, conditional on parental earnings deciles.

\subsection{Empirical implementation}

Assessing whether the Kindergarten Act equalized opportunities across Norwegian children requires two sets of outcome distributions: For each circumstance, the distribution of observed outcomes by family background among children who have experienced the childcare expansion, and the counterfactual distribution that would have prevailed in absence of the reform. Following Havnes and Mogstad (2015), we apply a difference-in-difference (DiD) approach, exploiting that the supply shocks to subsidized child care were larger in some areas than in others. Specifically, we compare the adult earnings of children aged 3 to 6 years old before and after the reform, from municipalities where child care expanded a lot (i.e. the treatment group) and municipalities with little or no increase in child care coverage (i.e. the comparison group).

We focus on the early expansion, which likely reflects the abrupt slackening of con-

\footnotetext{
${ }^{22}$ For detailed information about the program and for descriptive statistics, we refer the reader to Havnes and Mogstad (2011).
} 
straints on the supply side caused by the reform, rather than a spike in the local demand. We consider the period 1976-1979 as the child care expansion period. To define the treatment and comparison group, we order municipalities according to the percentage point increase in child care coverage rates over the expansion period. We then separate the sample at the median, letting the upper half be treatment municipalities and the lower half be comparison municipalities. To define the pre-reform and post-reform groups, we exploit that children born 1967-69 enter primary school before the expansion period starts, while children born 1973-76 are in child care age after the expansion period has ended. Havnes and Mogstad (2011, 2015) show that the expansion of child care is not explained by observable characteristics.

To assess the impact of the reform on the distribution of children's earnings, conditional on parental earnings, we estimate the following equation:

$$
\mathbb{1}\left\{y_{i t} \geq y\right\}=\gamma_{t}(y)+\left[\boldsymbol{\beta}_{\mathbf{0}}(y)+P_{t} \cdot \boldsymbol{\beta}_{\mathbf{1}}(y)+T_{i} \cdot \boldsymbol{\beta}_{\mathbf{2}}(y)+T_{i} \cdot P_{t} \cdot \boldsymbol{\beta}_{\mathbf{3}}(y)\right] \cdot \mathbf{x}_{i t}+\epsilon_{i t}(y),
$$

where $\mathbb{1}\{\cdot\}$ is the indicator function, $y_{i t}$ are average yearly earnings in 2006-2009 of child $i$ born in year $t$, and $y$ is a threshold value of earnings discussed below. $T_{i}$ is a dummy equal to one if the child is from a treatment municipality and zero otherwise, and $P_{t}$ is a dummy equal to one for post-reform cohorts (born 1973-76) and zero for pre-reform cohorts (born 1967-69). $\gamma_{t}$ is a birth cohort fixed effect, and $\epsilon$ is the error term. The vector $\mathbf{x}_{i t}$ contains a fourth-order polynomial in the average yearly earnings of the child's parents when the child was in child care age, that is $\mathbf{x}_{i t}^{\prime}=\left(x_{i t}, x_{i t}^{2}, x_{i t}^{3}, x_{i t}^{4}\right){ }^{23}$ Vectors $\boldsymbol{\beta}_{\mathbf{0}}(y), \boldsymbol{\beta}_{\mathbf{1}}(y), \boldsymbol{\beta}_{\mathbf{2}}(y)$ and $\boldsymbol{\beta}_{\mathbf{3}}(y)$ have dimension $(1 \times 4)$.

The vector $\boldsymbol{\beta}_{\mathbf{3}}(y)$ provides DiD-estimates of how the reform affected the earnings distribution of exposed children. In the spirit of standard DiD, the estimator uses the observed change in the distribution around the value $y$, from before to after treatment, as an estimate of the change that would have occurred in the treatment group over this period in the absence of treatment. The identifying assumption is that the change in population shares from before to after treatment around a given level of earnings would be the same in the treatment group as in the comparison group, in the absence of the treatment. 24

\footnotetext{
${ }^{23}$ We tested alternative polynomial specifications without any appreciable impact on results.

${ }^{24}$ The estimator may be regarded as a RIF-estimator, see Firpo, Fortin and Lemieux (2009) for a discussion. For a discussion of non-linear difference-in-differences methods, see Athey and Imbens (2006) or Havnes and Mogstad (2015).
} 
Note that equation (6) allows for heterogeneity in the effect of the reform on the distribution of earnings along two dimensions. First, $\boldsymbol{\beta}_{\mathbf{3}}(y)$ is a function of the threshold earnings so the effect of the reform is allowed to vary along the earnings distribution of the children. Second, since $\boldsymbol{\beta}_{\mathbf{3}}(y)$ is interacted with a polynomial in parental earnings $\left(\mathbf{x}_{i t}\right)$, the effect of the reform is allowed to vary according to family background.

Equation (6) provides estimates defined in terms of changes in probability mass at each value $y$. From these, we can compute the change in earnings induced by the reform by rescaling with an estimate of the density at $y$ (Firpo et al. 2009). When $y$ is a quantile, this yields an estimate of the quantile treatment effect (QTE).

Our EZOP criterion rests on a comparison of the effects of the reform at quantiles of the earnings distribution conditional on circumstances. For each circumstance $c$ and each quantile $p \in[0,1]$, define $Q_{1}(p \mid c)=F^{-1}(p \mid c, T=1, P=1)$ as the value of the $p^{t h}$ quantile in the actual distribution of earnings among treated children, conditional on circumstances. The estimated QTE at quantile $p$ for children with circumstances $c$ can then be defined as:

$$
Q T E(p \mid c)=\frac{\mathrm{E}\left[\boldsymbol{\beta}_{\mathbf{3}}\left(Q_{1}(p \mid c)\right) \cdot \mathbf{x}_{i t} \mid C_{i t}=c\right]}{f\left(Q_{1}(p \mid c) \mid C_{i t}=c\right)}
$$

where $C_{i t}$ denotes the circumstances of individual $i$ born in cohort $t$, and $f(\cdot \mid \cdot)$ denotes the density of the earnings distribution $F(\cdot \mid \cdot)$. Because $Q T E(p \mid c)$ estimates the impact of the treatment, we readily construct an estimate of the counterfactual quantile in the absence of treatment as $Q_{0}(p \mid c)=Q_{1}(p \mid c)-Q T E(p \mid c)$.

In the empirical application, we use the earnings decile of the child's parents to define circumstances. Parental earnings are used here as a catch-all measure of individual circumstances ${ }^{25}$ We estimate equation (6) using OLS at each percentile of the earnings distribution conditional on circumstances 26 We then use a kernel estimate of the density from this distribution to construct our estimate of $Q T E(p \mid c)$. Our estimation sample is based on Norwegian registry data and covers children born to married mothers, who constitute about $93 \%$ of the relevant cohorts. Standard errors are obtained using a non-

\footnotetext{
${ }^{25}$ While there are several other candidates to measure circumstances, these are typically strongly correlated with parental earnings. Indeed, Björklund et al. (2012) show that parental income per se is the most important characteristic among a large set of family circumstances. An advantage of our relatively simple measure is also that the circumstances have an immediately natural ranking, that would break down if interacted with e.g. parental education.

${ }^{26}$ In practice, we omit the bottom five percentiles to avoid issues of measurement error, and the top five percentiles to avoid problems arising from lack of overlap in the conditional distributions. We therefore run 90 regressions for each of the ten circumstances.
} 
parametric bootstrap with 300 replications. Based on our estimates of the actual and counterfactual outcome distributions and on the bootstrapped covariances, we implement stochastic dominance tests as discussed in the appendix.

\subsection{Results for three classes}

Defining children's circumstances from parental earnings deciles involves a large number of pairwise comparisons. To clarify the intuition behind the comparisons, we first focus on three types in the population: Children whose parents had earnings in the second, the fifth and the ninth decile, respectively. For expositional simplicity, we will refer to these simply as lower class, middle class and upper class children.

We start by analyzing the extent of inequality of opportunity before the implementation of the child care expansion. Panel (a) in figure 1 presents the counterfactual distributions $Q_{0}(p \mid c)$ that would have been observed in the absence of the policy $(\pi=0)$. The figure shows first order stochastic dominance when we compare any pair of distributions. This indicates that equality of opportunity is clearly violated. Furthermore, for all preferences, there is a clear ordering of family types, with upper class children doing better than middle class children, and middle class children doing better than lower class children.

Panel (b) in figure 1 shows the impact of the child care expansion on the earnings distribution of children in these three groups. The dashed line presents the QTE for middle class children. Overall, the effect of the child care expansion in this group is relatively modest. However, there is significant heterogeneity in the impact of the policy: within the middle class, effects are positive in the bottom of the earnings distribution, and turn negative in the upper end of the distribution. The dotted line gives the effect on upper class children. In this group, the reform has a modest positive impact for children in the bottom of the conditional distribution but a large negative impact in the top of the distribution. Finally, the solid line provides estimates of the effect of the child care expansion for lower class children. On average, lower class children seem to benefit more from the child care expansion than children from middle and upper class. Furthermore, the heterogeneity in the effect of child care stands in marked contrast with what was observed in the other two groups: Among lower class children, the reform had a small positive effect in the bottom of the distribution but had an increasingly large and positive effect as we move up the conditional earnings distribution. This suggests two likely conclusions. 
First, on average, child care appears substitutable with parental resources, captured here by the class of origin. Second, the impact of child care seems complementary to the child's idiosyncratic resources, within the lower class, while the opposite seems to be true in the middle and upper class.

Panel (c) of figure 1 presents the conditional distribution of earnings after the policy implementation $(\pi=1)$. The figure shows first order stochastic dominance when we compare any pair of distributions. Hence, equality of opportunity does not prevail, even after the implementation of the reform. However, compared to panel (a), the gap between any pair of curves seems to have fallen at almost every quantile of the earnings distribution, which suggests that the child care policy might have partially equalized opportunities across the three classes.

To implement our EZOP procedure, we present in panels (d)-(f) the estimated gap curves from pairwise comparisons of children from different family types under both social states, alongside gap curve differences between these states with a $99 \%$ confidence interval band. Since the conditional distributions can be ordered according to first order stochastic dominance, we may invoke proposition 2. Gap curve dominance provides a necessary and sufficient condition for equalization of opportunity.

Two main features stand out. First, in both social states, gap curves are virtually always positive. This reflects the fact that all groups are ordered according to stochastic dominance both with and without the child care reform. Second, the actual gap curve $(\pi=1)$ is almost always below the counterfactual gap curve $(\pi=0)$. This indicates that the reform reduced inequality of opportunity between all pairs of types. This fact is clarified by looking at the gap curve differences: While the difference is small and not statistically significant at the bottom of the distribution, the difference becomes positive and strongly statistically significant as we move up in the distribution.

The formal assessment of EZOP rests on joint tests of stochastic dominance, in each pairwise comparison of groups, for (i) the actual distributions, (ii) the counterfactual distributions and (iii) the gap curves. Results of these tests are presented in Table 1. Panel $\mathrm{A}$ and $\mathrm{B}$ present test statistics for the counterfactual and actual settings, respectively. We test three distinct hypotheses: the first is that distributions of the two groups are equal; the second is that the distribution of the underprivileged group first order stochastic dominates the distribution of the privileged group; the third is the reverse of the second hypothesis. 

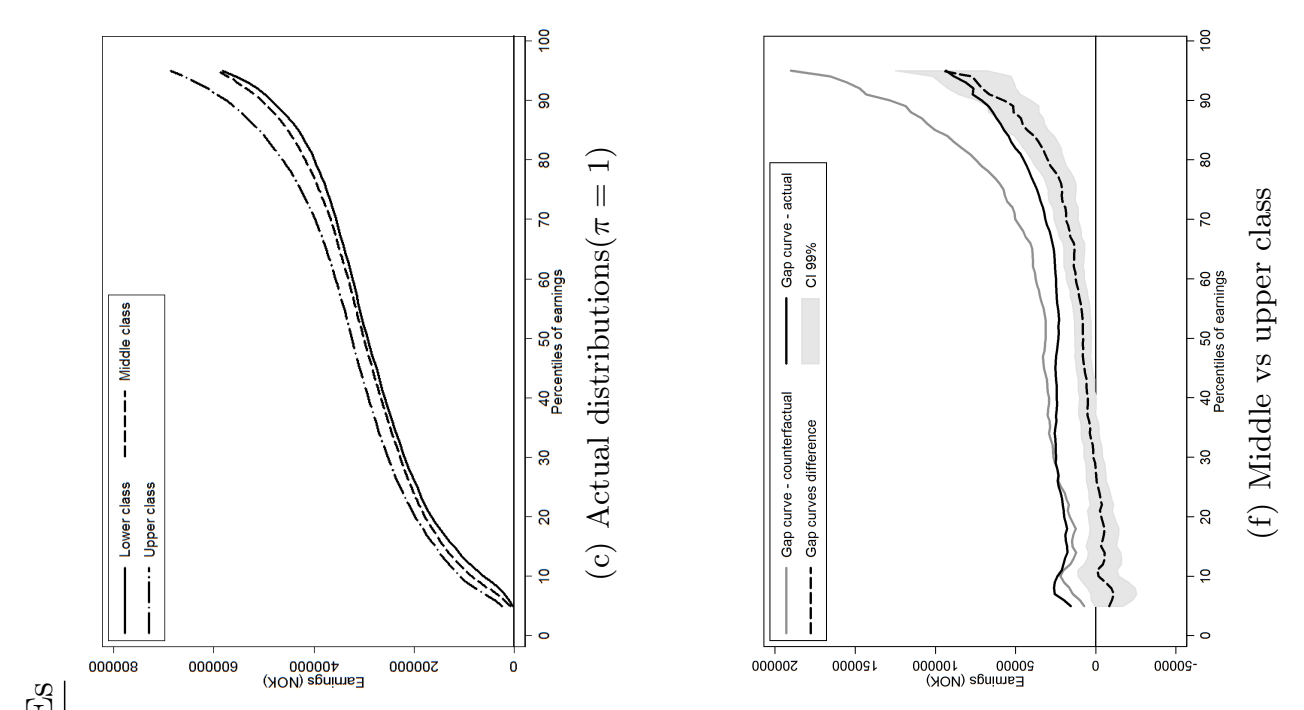

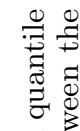
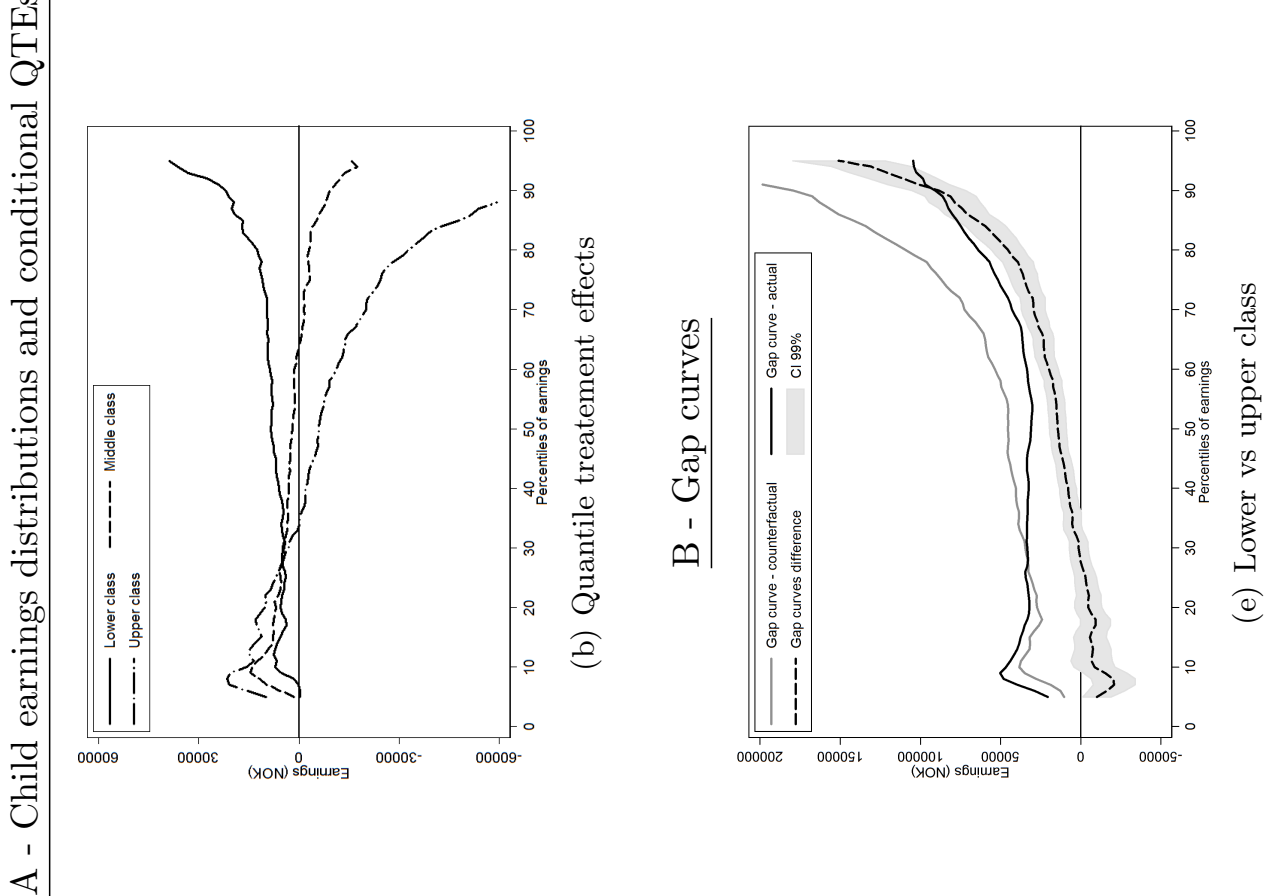

ฮี

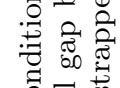

ำ

†

ॠ

눙

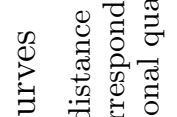

ठิ

赵赵

चี

先 0

幽

प क्षे

छ्व

Ð

\pm 氙

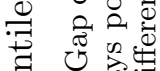

ప

की

:

苗

.⿹丁口

So

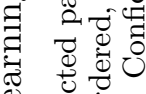

苍递宁

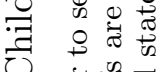
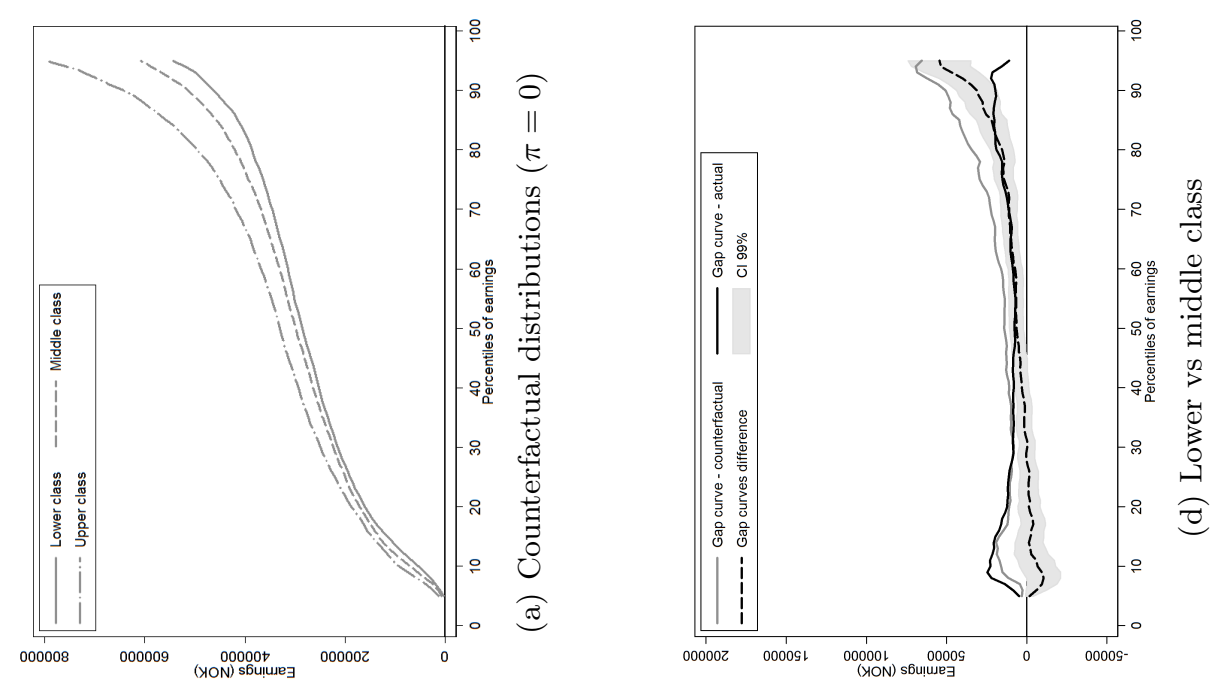

..

네

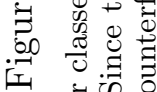

फ़े

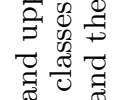

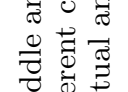

范苛

要击

웅

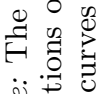

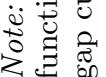


Table 1: Joint dominance and equality tests for actual and counterfactual children earning distributions and gap curves, for selected parental earnings deciles

\begin{tabular}{|c|c|c|c|c|c|c|}
\hline & \multicolumn{6}{|c|}{ Pairwise comparisons of social classes: } \\
\hline & \multicolumn{2}{|c|}{ Lower vs middle } & \multicolumn{2}{|c|}{ Lower vs upper } & \multicolumn{2}{|c|}{ Middle vs upper } \\
\hline \multicolumn{7}{|c|}{ A - Cdfs, counterfactual setting $(\pi=0)$} \\
\hline$H_{0}: \sim$ & 72.9 & {$[0.000]$} & 659.4 & $0.000]$ & 384.2 & $0.000]$ \\
\hline$H_{0}: \succcurlyeq$ & 72.9 & $0.000]$ & 659.4 & $0.000]$ & 384.2 & $0.000]$ \\
\hline$H_{0}: \preccurlyeq$ & 0.0 & $0.944]$ & 0.0 & $0.949]$ & 0.0 & $0.947]$ \\
\hline \multicolumn{7}{|c|}{ B - Cdfs, actual setting $(\pi=1)$} \\
\hline$H_{0}: \sim$ & 40.1 & $0.003]$ & 423.7 & $0.000]$ & 266.3 & $0.000]$ \\
\hline$H_{0}: \succcurlyeq$ & 40.1 & $0.000]$ & 423.7 & $0.000]$ & 266.3 & $0.000]$ \\
\hline$H_{0}: \preccurlyeq$ & 0.0 & $0.949]$ & 0.0 & $0.952]$ & 0.0 & $0.948]$ \\
\hline \multicolumn{7}{|c|}{ C - Gap curves $(\pi=0$ vs $\pi=1)$} \\
\hline$H_{0}:$ Neutrality & 84.2 & $0.000]$ & 266.4 & $0.000]$ & 125.0 & $0.000]$ \\
\hline$H_{0}:$ Equalization & 4.8 & $0.672]$ & 11.2 & $0.381]$ & 9.1 & $0.468]$ \\
\hline$H_{0}:$ Disequalization & 76.0 & $0.000]$ & 248.4 & $0.000]$ & 112.0 & $0.000]$ \\
\hline
\end{tabular}

Note: The table reports Wald-test statistics and associated $p$-values (in brackets) for various null hypothesis, comparing the earnings distribution of lower, middle and upper classes. In panels $\mathrm{A}$ and $\mathrm{B}$, for each of the three pairs of classes, we test the following three null hypothesis: equality of the cdfs $(\sim)$, first order stochastic dominance of the worse-off class over the well-off class $(\succcurlyeq)$ and first order stochastic dominance of the well-off class over the worse-off class $(\preccurlyeq)$. In panel C, for each pair of classes, we compare gap curves under the actual and counterfactual states and test three null hypothesis: the gap curves are statistically equal (neutrality); the gap curve in the counterfactual policy state is everywhere larger than in the actual policy state (equalization); the gap curve in the counterfactual policy state is everywhere smaller than in the actual policy regime (disequalization). Gap curves are defined according to the order of groups estimated from panels A and B. Covariances are bootstrapped. Joint tests are performed on ventiles of child earnings distributions. In panels $\mathrm{A}$ and $\mathrm{B}$ of table 1 the values of the tests statistics taken under the null hypothesis of equality and dominance coincide. This is a consequence of the definition of the test statistics presented in the appendix.

Not surprisingly, only the third hypothesis cannot be rejected in all comparisons.

Finally, panel C presents the main tests of equalization of opportunity. First, the null hypothesis is that the reform had no impact on inequality of opportunity (neutrality). This hypothesis is strongly rejected by the data. Second, the null hypothesis is that the reform equalized outcomes across children from different classes (equalization). This hypothesis cannot be rejected by the data, with $p$-values above 0.38 . Third, the null hypothesis is that the reform disequalized outcomes, increasing inequality of opportunity. In this case, we can again strongly reject the hypothesis in all comparisons.

To summarize, the analysis shows first that the ordering between children from different classes in terms of their labor market performance is quite clear in Norway: Upper class children dominate middle class children who dominate lower class children. Second, the analysis shows that the child care reform in 1975 did indeed equalize substantially the opportunities across children from different classes. Using the Gini-type evaluation function, we can quantify the effect of the policy. For low and middle classes, results indicate that the reform had a positive effect: Their opportunities increased by $4.3 \%$ and $3 \%$, re- 
spectively. In contrast, the value of the opportunity set of the upper class increased only by a modest $1 \%$, which turns out to be statistically insignificant. This differential in growth rates indicates that the lower and middle classes benefited from the policy reform, both in absolute and in relative terms, in the sense that they caught up with the upper class ${ }^{27}$ Third, the QTE estimates show that this equalization came both from positive impacts at the lower end of the distribution and from negative impacts at the upper end for many children. This raises a concern about the universal design of the child care expansion, as discussed in Havnes and Mogstad (2015).

\subsection{Results for all parental earnings deciles}

We now consider the entire population of children and extend the above group-comparisons to all ten deciles of the parental earnings distribution. The results of the same series of tests as in table 1 are summarized graphically in figure 2. In each panel, colored squares summarize the results of the tests of the hypothesis of dominance of the groups on the vertical axis over the groups on the horizontal axis. The shading of the squares indicates the $p$-value for the rejection of the null hypothesis of dominance. Dark squares indicate failure to reject the null hypothesis of dominance (i.e. high $p$-values), while light squares indicate rejection of the null hypothesis. We also test for equality of the distributions across groups, and indicate failure to reject equality with a black bullet inside the square 28

Panels A and B of figure 2 report the results of dominance tests in the counterfactual and in the actual states. In both states, the results suggest a strong monotonic relation between parental earnings and the earnings advantage of children. Above the diagonal, we universally fail to reject the hypothesis that the earnings distribution of children from higher parental deciles dominates that of children from lower parental deciles. Below the diagonal, we do reject that the earnings distribution of lower-decile children dominates that of higher-decile children virtually everywhere. The only exceptions are three central

\footnotetext{
${ }^{27}$ The difference and bootstrapped t-statistics (in parenthesis) between lower and middle classes are 0.0073 (3.091); between lower and upper classes is 0.0206 (3.185) and between middle and upper classes is 0.0133 (2.693).

${ }^{28}$ To illustrate the construction of the figure, compare with the analysis with only three classes, in the previous section. The squares in row 2 , column 9 , and in row 9, column 2, compare children from lower class (D2) to children from upper class (D9). These squares represent p-values for the joint tests in the central column of table 1. Consider panel A of figure 2 where we test for dominance in the counterfactual setting. The dark color in row 9, column 2 indicates the failure to reject dominance in the third line of panel A in table 1. Similarly, the light color in row 2, column 9 indicates the rejection of dominance in the second line of panel A in table 1 The absence of bullets in both blocks indicates that we reject equality of the earnings distributions, as in the first line of panel A in table 1 .
} 

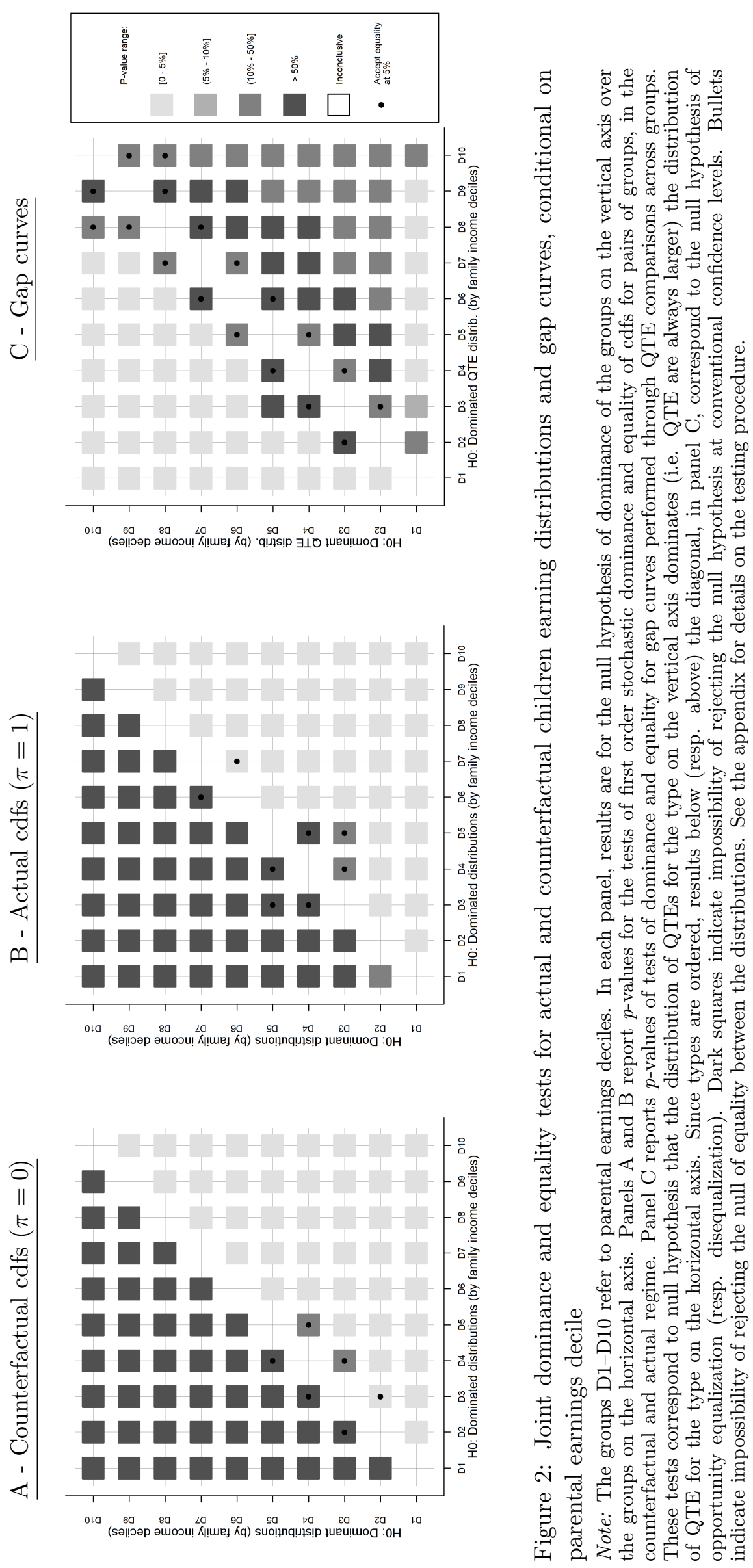
comparisons around the diagonal, where the differences in parental earnings across groups is rather small, and equality cannot be rejected. Overall, these tests provide clear evidence of inequality of opportunity for earnings among Norwegian children in both states.

We now turn to the test of equalization of opportunity. Panel $\mathrm{C}$ of figure 2 reports the results of gap curve dominance tests for all pairs. For two thirds of the comparisons (29 out of 45) we find the following pattern: Below the diagonal, we do not reject an improvement in the position of the less advantaged children compared to more advantaged children. Above the diagonal, we do reject an improvement in the position of the more advantaged children compared to less advantaged children. Hence, these results indicate that, in most pairwise comparisons, the implementation of the policy significantly decreases the opportunity gap between the advantaged and the disadvantaged type.

There are, however, two main exceptions. For ten pairs we fail to reject both equalization and disequalization 29 Furthermore, we also find that the gap curves are statistically equal before and after, which indicates that the policy left inequality of opportunity unchanged. Thus, for these pairs, the condition of proposition 2 is also weakly satisfied. The second exception is the comparison of group D1 to groups D3 to D9. In these cases, we reject both the hypothesis of equalization and the hypothesis of disequalization of opportunity. The tests are thus inconclusive: We cannot find evidence of gap dominance in any direction.

To summarize, we find that pairwise equalization of opportunity is satisfied in $85 \%$ of cases. However, most of the comparisons involving D1 are inconclusive, as we can conclude neither in favor of equalization nor disequalization. Taken together, the condition stated in proposition 5 is not satisfied for first order stochastic dominance. To overcome this lack of unanimous judgment on equalization of opportunity, we can follow two alternative routes. First, we may resort to a specific inequality of opportunity index. Using the Gini Opportunity index of Lefranc et al. (2008), we find that unfair inequality decreased by $8.8 \%$ as a result of the expansion in kindergarten provision 30 This comes as no surprise considering the large number of pairs where equalization of opportunity is found.

This does not, however, provide a satisfactory answer to the inconclusive results for group D1. This inconclusiveness arises from the fact that gap curves intersect. To go

\footnotetext{
${ }^{29}$ The pairs are all the adjacent pairs except those involving D1, and the pair D3-D5 and D8-D10 pairs.

${ }^{30}$ The Gini Opportunity indices (standard error) are $G O(0)=0.0358(0.0013)$ and $G O(1)=0.0326$ (0.001). The $p$-value for $H_{0}: G O(0)=G O(1)$, based on bootstrapped standard errors, is 0.029 .
} 


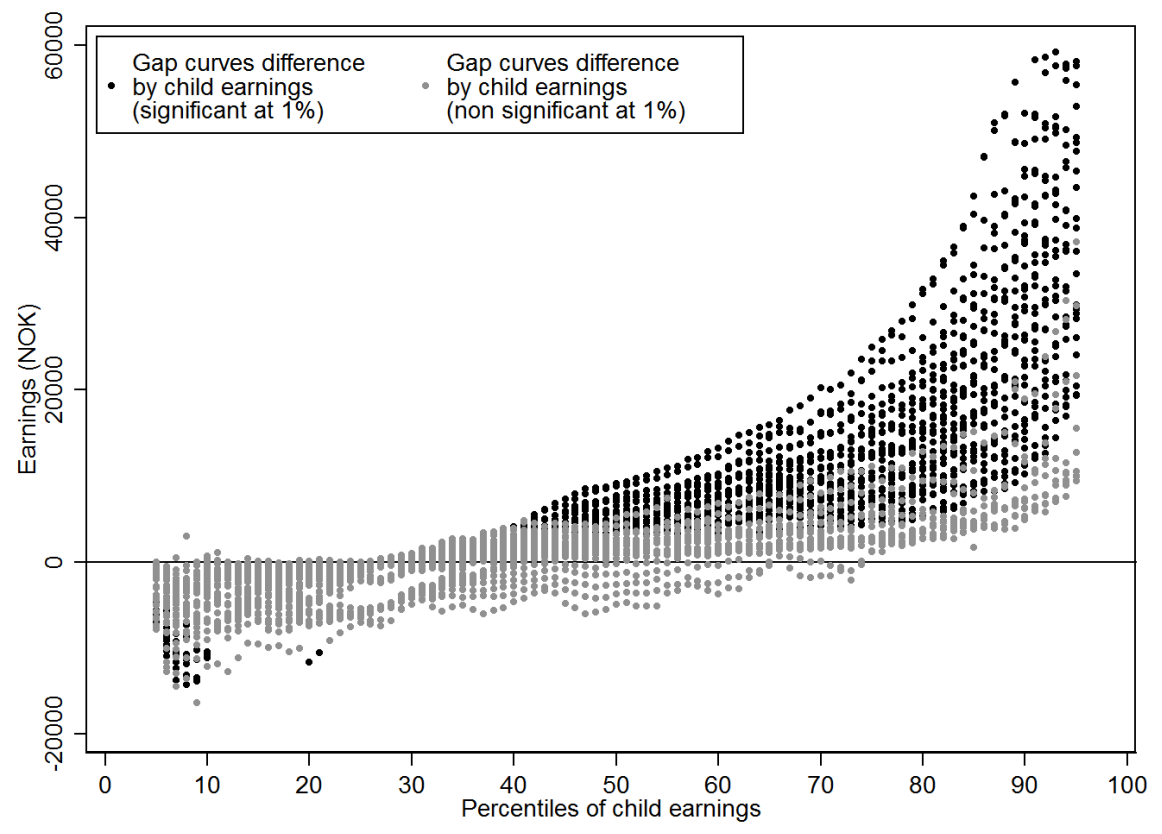

Figure 3: Equalization of opportunity across parental earnings deciles.

Note: The figure reports, for every percentile of the sons earnings distribution, the differences in QTE associated to pairs of different groups, i.e. the difference between the gap curves of the two types. Disjoint tests of equality of the QTE are performed using bootstrapped standard errors. Differences in QTE that are statistically indistinguishable from zero are reported in gray. Groups are ordered according to ISD at order one. Out of 10 groups of parental earnings, 45 pairs of comparisons are performed at every percentiles of the sons earnings distribution.

beyond, we may investigate the existence of higher order dominance as discussed above. In our data, we find that the integrated gap curve of order 3 before the policy is dominated by the gap curve after the policy in the comparison of group D1 to groups D3-D9. This implies that for all preferences in class $\mathcal{R}^{3}$, the child care reform caused disequalization of opportunity for the most disadvantaged group. The details of this analysis are in the appendix.

Lastly, it is worth analyzing what parts of the distribution contribute to changes in the gap between pairs of groups. To clarify this point, figure 3 reports the difference between gap curves in the actual and counterfactual state, for each percentile of the children's earnings distribution. At each percentile of the conditional earnings distributions of the children, we report the difference between the gap curves at that percentile from each of the 45 comparisons below the diagonal in panel $\mathrm{C}$ of figure2. Black dots indicate gap curve differences that are statistically significant at the $1 \%$ level. Figure 3 shows that equalization among most of the groups is driven not by a reduction in the gap at the bottom end of the children's distributions, but rather by a narrowing of the gap in the middle and upper end 
of the distributions. This is explained partly by the fact that estimated effects are rather homogenous across groups at the lower end of the distribution, and partly by the fact that the negative QTE-estimates at the upper end of the distribution are particularly large for advantaged groups.

\section{Concluding remarks}

The first contribution of this paper is theoretical. We develop a new criterion for ranking social states from the equality of opportunity perspective. Our criterion for equalization of opportunity entails a difference-in-differences comparison of outcome distributions conditional on circumstances. First, types are compared within each social state separately, to assess the direction and distribution of unfair advantage across all possible pairs of types. Second, differences are taken between social states in order to assess changes in the extent and distribution of unfair advantage.

We propose an innovative model based on comparisons of changes in the economic distance between pairs of distributions. Our criterion requires unanimity, within a large class of preferences, in the evaluation of the fall in the illegitimate advantage enjoyed by one type with respect to another. We study identification procedures and implementation issues, showing the equivalence of our EZOP order with gap curve dominance. In cases where the ordering of types is not unanimous, we proceed by minimally refining the set of potential preferences until agreement is reached. We show that this refinement is easily implementable using inverse stochastic dominance tools.

Our results extend to the equality of opportunity framework some important results in social welfare ordering. Several authors have demonstrated the equivalence between stochastic dominance orders and social orders in a welfarist context (Kolm 1969, Atkinson 1970, Shorrocks 1983, Aaberge et al. 2014). Instead of focusing on inequality of outcomes, as in the welfarist approach, our social order criterion is based on the principle of equality of opportunity, in line with modern theories of distributive justice and extends this approach.

Our second contribution is to provide a statistical framework that allows to implement our equalization of opportunity criterion. Our application also underlines that econometric models allowing for heterogenous effects can be tightly connected to the normative assessment of distributional issues. The recent econometric literature has provided important 
tools for estimating the heterogenous impact of policy intervention on some outcome of interest ${ }^{31}$ Since our equalization criterion can be expressed in terms of restrictions on quantile treatment effects, this paper suggests a simple way in which these estimates can be used to assess whether a given policy helps to promote distributive justice.

The third contribution of this paper pertains to the empirical analysis of the effectiveness of early childhood intervention at equalizing life chances. Growing evidence on the role of family background on lifelong earnings potential (Björklund and Salvanes 2011, Black and Devereux 2010) has brought educational policies to the forefront as potential tools for alleviating differences stemming from family background. This has taken particular prominence due to theory and evidence suggesting that skills formation early in life may be crucial in determining children's trajectories (Cunha and Heckman 2007). Expanding access to quality child care may be expected to equalize opportunities among treated children, by weakening the dependence between family background and children's development. While studies of targeted programs often find positive effects (for a survey, see e.g. Blau and Currie 2006), the literature on universal programs is smaller and findings are mixed (see Havnes and Mogstad (2015) and references therein). We extend this literature by providing evidence on the impact of a universally available large scale child care program on long run equality of opportunity.

Applying our framework to evaluate the introduction of universally available child care in Norway, we conclude that kindergarten expansion indeed equalizes opportunities among children from most family backgrounds. Two important caveats should be noted. First, echoing results in Havnes and Mogstad (2015), our results show that the equalization of opportunity resulting from the reform is driven importantly by reduced earnings at the upper end of the earnings distribution for affected children. An important question is whether resources devoted to provide child care for children from upper class families could be reallocated to improve quality or uptake of child care for lower class children.

Second, although there is strong agreement on equalization of opportunity for the vast majority of groups, it is not possible to conclude completely in favor of equalization. In fact, the policy seems to increase the gap for the least successful children in the most disadvantaged group compared to most other types. This result indicates that the Kindergarten

\footnotetext{
${ }^{31}$ Abadie, Angrist and Imbens (2002), Chernozhukov and Hansen (2005), Athey and Imbens (2006) and Firpo et al. (2009) are important contributions to this literature. The RIF-DiD estimator of Havnes and Mogstad (2015) belongs to the same econometric vein.
} 
Act produced relatively low returns for these children, leaving them even further behind compared to the children from somewhat less disadvantaged backgrounds, that benefitted handsomely. This finding casts a shadow on the effectiveness of universal child care for the neediest children and deserves further investigation.

\section{References}

Aaberge, R. (2009). Ranking intersecting Lorenz curves, Social Choice and Welfare 33: $235-259$.

Aaberge, R., Havnes, T. and Mogstad, M. (2014). A theory for ranking distribution functions. UiO Memo 20/2014.

Abadie, A., Angrist, J. and Imbens, G. (2002). Instrumental variables estimates of the effect of subsidized training on the quantiles of trainee earnings, Econometrica 70(1): $91-117$.

Allais, M. (1953). Le comportement de l'homme rationnel devant le risque, critique des postulates et axiomes de l'ecole américaine, Econometrica 21: 503-546.

Almond, D. and Currie, J. (2011). Human Capital Development before Age Five, Vol. 4 of Handbook of Labor Economics, Elsevier, chapter 15, pp. 1315-1486.

Almås, I., Cappelen, A. W., Lind, J. T., Sørensen, E. . and Tungodden, B. (2011). Measuring unfair (in)equality, Journal of Public Economics 95(7-8): 488-499.

Athey, S. and Imbens, G. W. (2006). Identification and inference in nonlinear differencein-differences models, Econometrica 74(2): pp. 431-497.

Atkinson, T. B. (1970). On the measurement of inequality, Journal of Economic Theory 2: $244-263$.

Baker, M. (2011). Innis lecture: Universal early childhood interventions: what is the evidence base?, Canadian Journal of Economics 44(4): 1069-1105.

Björklund, A., Jäntti, M. and Roemer, J. (2012). Equality of opportunity and the distribution of long-run income in sweden, Social Choice and Welfare 39(2): 675-696.

Björklund, A. and Salvanes, K. G. (2011). Education and Family Background: Mechanisms and Policies, Vol. 3 of Handbook of the Economics of Education, Elsevier, chapter 3, pp. 201-247.

Black, S. E. and Devereux, P. J. (2010). Recent developments in intergenerational mobility, in D. Card and O. Ashenfelter (eds), Handbook of Labor Economics, Vol. 4B, NorthHolland, Amsterdam, chapter 8.

Blau, D. and Currie, J. (2006). Pre-School, Day Care, and After-School Care: Who's Minding the Kids?, Vol. 2 of Handbook of the Economics of Education, Elsevier, chapter 20, pp. 1163-1278. 
Bourguignon, F., Ferreira, F. H. G. and Menendez, M. (2007). Inequality of opportunity in Brasil, Review of Income and Wealth 53(4): 585-618.

Chakravarty, S. R. (1988). Extended Gini indices of inequality, International Economic Review 29(1): 147-156.

Chakravarty, S. R. and Dutta, B. (1987). A note on measures of distance between imcome distributions, Journal of Economic Theory 41(1): 185 - 188.

Checchi, D. and Peragine, V. (2010). Inequality of opportunity in Italy, Journal of Economic Inequality 8: 429-450.

Chernozhukov, V. and Hansen, C. (2005). An IV Model of Quantile Treatment Effects, Econometrica 73(1): 245-261.

Cunha, F. and Heckman, J. (2007). The technology of skill formation, American Economic Review 97(2): 31-47.

Dworkin, R. (1981). What is equality? Part 1: Equality of welfare, Philosophy $\&$ Public Affairs 10(3): pp. 185-246.

Ferreira, F. H. G. and Gignoux, J. (2011). The measurement of inequality of opportunity: Theory and an application to latin america, Review of Income and Wealth $\mathbf{5 7}(4)$ : 622657 .

Ferreira, F. H. G. and Peragine, V. (2016). Individual Responsibility and Equality of Opportunity, Vol. The Oxford Handbook of Well-Being and Public Policy, Oxford University Press, chapter 25.

Firpo, S., Fortin, N. M. and Lemieux, T. (2009). Unconditional quantile regressions, Econometrica 77(3): 953-973.

Fleurbaey, M. (2008). Fairness, Responsability and Welfare, Orxford University Press.

Fleurbaey, M. and Peragine, V. (2013). Ex ante versus ex post equality of opportunity, Economica 80(317): 118-130.

Havnes, T. and Mogstad, M. (2011). No child left behind: Subsidized child care and children's long-run outcomes, American Economic Journal: Economic Policy 3: 97129 .

Havnes, T. and Mogstad, M. (2015). Is universal child care leveling the playing field?, Journal of Public Economics 127: 100-114.

Heckman, J. J. and Masterov, D. V. (2007). The productivity argument for investing in young children, Working Paper 13016, National Bureau of Economic Research.

Kahneman, D. and Tversky, A. (1979). Prospect theory: An analysis of decision under risk, Econometrica 47: pp. 263-293.

Kolm, S.-C. (1969). The Optimal Production of Social Justice, Public Economics, London: McMillan, pp. 145-200.

Lefranc, A., Pistolesi, N. and Trannoy, A. (2008). Inequality of opportunities vs. inequality of outcomes: Are western societies all alike?, Review of Income and Wealth 54(4): 513546 . 
Lefranc, A., Pistolesi, N. and Trannoy, A. (2009). Equality of opportunity and luck: Definitions and testable conditions, with an application to income in France, Journal of Public Economics 93(11-12): 1189 - 1207.

Lefranc, A. and Trannoy, A. (2017). Equality of opportunity, moral hazard and the timing of luck, Social Choice and Welfare (forthcoming).

Moyes, P. (1987). A new concept of Lorenz domination, Economics Letters 23(2): 203 207.

Muliere, P. and Scarsini, M. (1989). A note on stochastic dominance and inequality measures, Journal of Economic Theory 49(2): 314 - 323.

O’Neill, D., Sweetman, O. and Van De Gaer, D. (2000). Equiality of opportunity and kernel density estimation: An application to intergenerational mobility, Advances in Econometrics : A research Annual 14: 259-274.

Peragine, V. (2002). Opportunity egalitarianism and income inequality, Mathematical Social Sciences 44(1): $45-64$.

Quiggin, J. (1981). Risk perception and risk aversion among australian farmers, Australian Journal of Agricultural Economics 25(2): 160-169.

Ramos, X. and Van de gaer, D. (2016). Approaches to inequality of opportunity: Principles, measures and evidence, Journal of Economic Surveys 30(5): 855-883.

Roemer, J. (1998). Equality of Opportunity, Harvard University Press, Cambridge.

Roemer, J. and Trannoy, A. (2014). Equality of opportunity, Vol. Handbook of Income Distribution.

Ruhm, C. J. and Waldfogel, J. (2012). Long-term effects of early childhood care and education, Forthcoming in Nordic Economic Policy Review .

Sen, A. (1974). Informational bases of alternative welfare approaches : Aggregation and income distribution, Journal of Public Economics 3(4): 387-403.

Shorrocks, A. F. (1983). Ranking income distributions, Economica 50(197): pp. 3-17.

Van de gaer, D. (1993). Equality of Opportunity and Investment in Human Capital, PhD thesis, Katholieke Universiteit Leuven.

Weymark, J. A. (2003). Generalized Gini indices of equality of opportunity, Journal of Economic Inequality 1: 5-24.

Yaari, M. E. (1987). The dual theory of choice under risk, Econometrica 55(1): pp. 95-115.

Zoli, C. (2002). Inverse stochastic dominance, inequality measurement and Gini indices, Journal of Economics 9: 119-161. 


\title{
Appendix for online publication
}

\author{
Robust inequality of opportunity comparisons: \\ Theory and application to early-childhood policy evaluation
}

\author{
Francesco Andreoli ${ }^{\mathrm{a},}$, , Tarjei Havnes ${ }^{\mathrm{b}}$, and Arnaud Lefranc ${ }^{\mathrm{c}}$ \\ ${ }^{a}$ LISER - Luxembourg Institute of Socio-Economic Research \\ ${ }^{\mathrm{b}}$ University of Oslo \\ ${ }^{\mathrm{c}}$ THEMA, University of Cergy-Pontoise and IZA
}

June 30,2017

\section{A Definitions and proofs}

\section{A.1 Notions of stochastic dominance}

Consider the following transformation of the inverse cumulative distribution function $F^{-1}(p)$, indexed by the natural number $k$ :

$$
\Lambda^{k}(p)=\int_{0}^{p} \Lambda^{k-1}(t) d t \text { and } \Lambda^{2}(p)=\int_{0}^{p} F^{-1}(t) d t
$$

where $\Lambda^{2}(p)$ is the generalized Lorenz curve introduced by Gastwirth (1971). The distribution $F(y)$ inverse stochastic dominates $F^{\prime}(y)$ at order $k$, denoted $F(y) \succcurlyeq_{I S D k} F^{\prime}(y)$, if and only if $\Lambda^{k}(p) \geq \Lambda^{\prime k}(p)$ for all $p \in[0,1]$, where the inequality holds strict at least for some values of $p$. At order $k=1$, ISD1 and stochastic dominance coincide. At order $k=2$, ISD2 and generalized Lorenz dominance coincide. For $k \geq 3$, inverse and direct stochastic dominance orders differ. The ISD $k$ criterion induces a partial ranking of distributions: when the curves representing $\Lambda^{k}(p)$ and $\Lambda^{\prime k}(p)$ cross in at least one point, the two distributions $F$ and $F^{\prime}$ cannot be ordered.

Following Muliere and Scarsini (1989) and Aaberge, Havnes and Mogstad (2014) it can be established that ISD $k$ is a necessary and sufficient condition for agreement over the preferred distribution among all preferences in $\mathcal{R}^{k}$, the set of rank-dependent utility functions where sign restrictions have been placed on the derivatives (up to order $k$ ) of the

${ }^{*}$ Corresponding author: LISER, 11 Porte des Sciences, Esch-sur-Alzette, L-4366, Luxembourg. E-mails: francesco.andreoli@liser.lu (F. Andreoli), tarjei.havnes@econ.uio.no (T.Havnes) and arnaud.lefranc@u-cergy.fr (A. Lefranc). 
underlying weighting functions. Furthermore, Maccheroni, Muliere and Zoli (2005) show that if $F_{\pi}(y \mid c, e) \succcurlyeq_{I S D k} F_{\pi}\left(y \mid c^{\prime}, e\right)$ then $F_{\pi}(y \mid c, e) \succcurlyeq_{I S D l} F_{\pi}\left(y \mid c^{\prime}, e\right)$, for all $l>\left.k\right|^{1}$

We now show that there always exists a degree $k$ at which any pair of distributions can be compared according to ISD ${ }^{2}$ The dominating distribution is the one that grants higher incomes to the poorest quantiles.

Proposition 1 For any pair of distributions with bounded support, with inverse cumulative distribution functions denoted by $F^{-1}($.$) and F^{\prime-1}($.$) , if \exists p_{\beta}>0 \mid \forall p \in\left[0, p_{\beta}\right) F^{-1}(p) \geq$ $F^{\prime-1}(p)$ and the strict inequality holds on a positive mass interval $\left[p_{\beta}-\epsilon, p_{\beta}\right)$ with $\epsilon>0$, we have:

$\exists k^{*} \in \mathbb{R}_{+}$and finite such that $F \succ_{I S D k} F^{\prime} \forall k \in \mathbb{N}_{+}$such that $k>k^{*}$.

Proof. Using the gap curve notation, we define $\Gamma^{k}(p):=\Gamma^{k}\left(F, F^{\prime}, p\right)=\Lambda^{k}(p)-\Lambda^{\prime k}(p)$ and $\Gamma(p):=F^{-1}(p)-F^{-1}(p)$ at any $p \in[0,1]$. Integrating by parts the function $\Gamma^{k}(p)$, up to $k-2$ times, gives:

$$
\begin{aligned}
\Gamma^{k}(p) & =\int_{0}^{p} \Gamma^{k-1}(t) d t=-\int_{0}^{p} t \cdot \Gamma^{k-2}(t) d t+\left[t \Gamma^{k-1}(t)\right]_{0}^{p} \\
& =\int_{0}^{p}(p-t) \Gamma^{k-2}(t) d t \\
& =\int_{0}^{p} \frac{1}{2}(p-t)^{2} \Gamma^{k-3}(t) d t+\left[\frac{1}{2}(p-t)^{2} \Gamma^{k-2}(t)\right]_{0}^{p} \\
& =\int_{0}^{p} \frac{1}{(k-2) !}(p-t)^{k-2} \Gamma(t) d t
\end{aligned}
$$

The result in 11 follows from the fact that $\Lambda^{k}(0)=0$ and therefore $\Gamma^{k}(0)=0$ for any $k$, and that $\Gamma^{2}(p)=\int_{0}^{p} \Gamma(t) d t$.

Let assume from the outset that $F$ should be the dominating distribution. If it is the case, there must exist a point $p_{\beta} \in[0,1]$ such that $\Gamma(p) \geq 0$ for all $p \in\left[0, p_{\beta}\right]$ with possibly some strong inequalities. Moreover, in the worst case $\Gamma(p) \leq 0$ for all $p \in\left(p_{\beta}, 1\right]$. If $\Gamma(p)=0$ for all $p \in\left[0, p_{\beta}\right]$, then by definition $F^{\prime} \succcurlyeq_{I S D 1} F$. Furthermore, since $F$ and $F^{\prime}$ are left continuous and bounded, then $\Gamma(p)$ is well behaved and takes only finite values on $p=1$ and $p=0$. Though not necessary, this assumption simplifies some technicalities.

This discussion clarifies that the upper bound (denoted $\bar{\alpha}>0$ ) and lower bound (denoted $-\beta>0$ ) of $\Gamma(p)$ should exists, as illustrated in figure 1. Formally: $\bar{\alpha}:=\sup \{\Gamma(p)$ : $\left.p \in\left[0, p_{\beta}\right)\right\}>0$ and $-\beta:=\inf \left\{\Gamma(p): p \in\left(p_{\beta}, 1\right]\right\}<0$.

Consider a scalar $\alpha \in(0, \bar{\alpha}]$ defining at least two points $p_{\alpha}, p_{\alpha}^{\prime} \in\left[0, p_{\beta}\right)$, such that $\Gamma(p)>0$ for all $p \in\left[p_{\alpha}, p_{\alpha}^{\prime}\right]$. This allows to define a new curve, denoted $\widetilde{\Gamma}(p)$ and repre-

\footnotetext{
${ }^{1}$ It is well known (e.g. Muliere and Scarsini 1989) that first and second order inverse stochastic dominance are equivalent to direct first and second order stochastic dominance, which is implemented by generalized Lorenz dominance when means coincide (Shorrocks 1983). Atkinson (1970) showed the logical relation between $G L$ dominance with fixed means and an the utilitarian social welfare function, later generalized to all $S$-concave social welfare functions and to income distributions with different means.

${ }^{2}$ This result holds for distributions with bounded support. It is connected to the result in Aaberge et al. (2014) that, as $k$ goes to infinity, order $k$ inverse stochastic dominance comparisons amount to a comparison of the lowest income in each distribution with bounded support.
} 


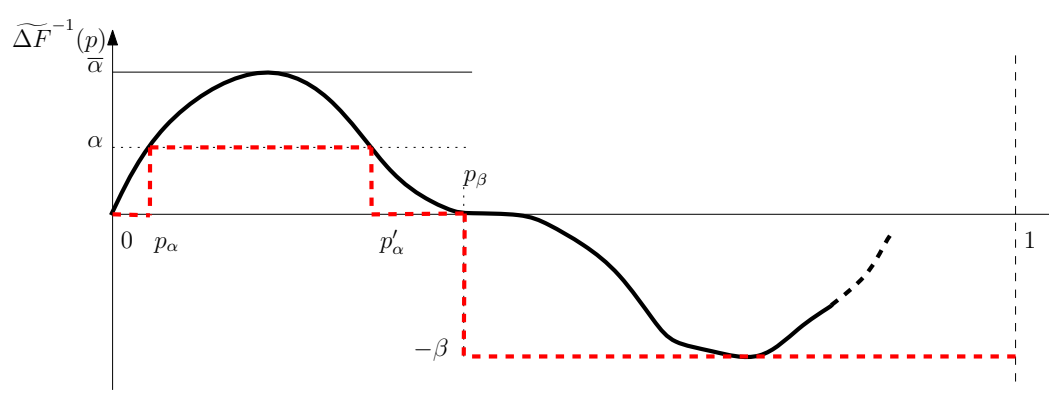

Figure 1: Proof of Proposition 1, The curves $\Gamma(p)$ (solid black) and $\widetilde{\Gamma}(p)$ (dashed red)

sented by a dashed line in figure 1. The equation of this curve is:

$$
\widetilde{\Gamma}(p):= \begin{cases}0 & \text { if } p \in\left[0, p_{\alpha}\right) \\ \alpha & \text { if } p \in\left[p_{\alpha}, p_{\alpha}^{\prime}\right] \\ 0 & \text { if } p \in\left(p_{\alpha}^{\prime}, p_{\beta}\right) \\ -\beta & \text { if } p \in\left[p_{\beta}, 1\right]\end{cases}
$$

The points $\alpha$ and $-\beta$ are identified by a given gap curve $\Gamma(p)$, so it always holds by construction that $\widetilde{\Gamma}(p) \leq \Gamma(p)$ for all $p \in[0,1]$. This is because $\widetilde{\Gamma}(p)$ reduces to the minimum the positive part of $\Gamma(p)$ while it magnifies the part where the curve takes on negative values. As a consequence, any recursive integral of $\widetilde{\Gamma}(p)$ will always lie above the corresponding integral of $\Gamma(p)$. To prove the proposition, it is therefore sufficient to show that, given the premises, there exists an order of integration of $\widetilde{\Gamma}(p)$ for which the resulting curve is always non-negative for any $p \in[0,1]$. Denote a real positive $\widetilde{k}^{*}$ such that $\forall k>\widetilde{k}^{*}$ :

$$
\int_{0}^{p} \frac{1}{(k-2) !}(p-t)^{k-2} \widetilde{\Gamma}(t) d t \geq 0 \quad \forall p \in[0,1]
$$

then, there must exists also a real positive value $k^{*}$ for which the proposition is true.

Noticing that the expression in 22 can take on non-zero values only on the interval $\left[p_{\alpha}, p_{\alpha}^{\prime}\right]$ and negative on the interval $\left[p_{\beta}, 1\right]$, it can be simplified as follows:

$$
\begin{aligned}
\int_{0}^{p} \frac{(p-t)^{k-2}}{(k-2) !} \widetilde{\Gamma}(t) d t & =\frac{1}{(k-2) !}\left[\int_{p_{\alpha}}^{p_{\alpha}^{\prime}}(p-t)^{k-2} \alpha d t+\int_{p_{\beta}}^{p}(p-t)^{k-2}(-\beta) d t\right] \\
& =\frac{\left\{\alpha\left[\left(p-p_{\alpha}\right)^{k-1}-\left(p-p_{\alpha}^{\prime}\right)^{k-1}\right]-\beta\left(p-p_{\beta}\right)^{k-1}\right\}}{(k-2) !} \geq 0 .
\end{aligned}
$$

To check the validity of the proposition, it is necessary to verify that the above expression is true for any $p \geq p_{\beta}$ only. To show this, it is sufficient to show that there exists a $\widetilde{k}^{*}$ such that:

$$
\frac{\left(p-p_{\alpha}\right)^{k-1}-\left(p-p_{\alpha}^{\prime}\right)^{k-1}}{\left(p-p_{\beta}\right)^{k-1}} \geq \frac{\beta}{\alpha}, \quad \forall p \geq p_{\beta}
$$

By construction of $\widetilde{\Gamma}(p)$, if the condition holds for $p=1$, then it must hold for all $p<1$, because the differential takes only negative values for $p \geq p_{\beta}$. Note that the numerator and denominator of the left hand side of (3) are positive, but the ratio is not said to be greater than one. Nevertheless, one can always find a value of $\alpha<\bar{\alpha}$ such that $\left(p-p_{\alpha}^{\prime}\right) \approx\left(p-p_{\beta}\right)$ 


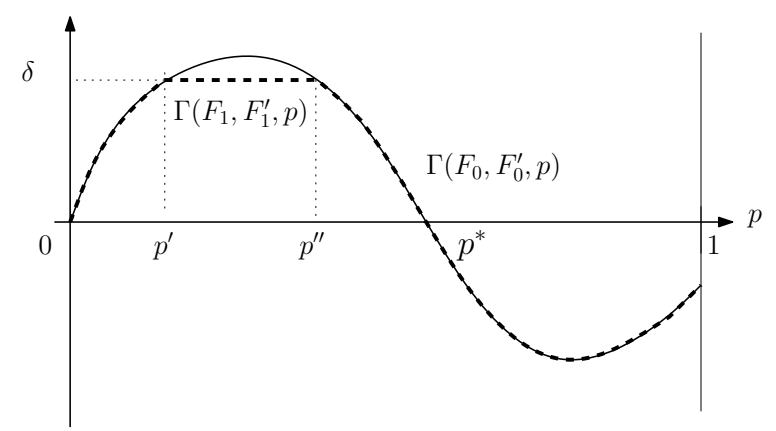

Figure 2: The curves $\Gamma\left(F_{0}, F_{0}^{\prime}, p\right)$ (solid line) and the perturbation generating $\Gamma\left(F_{1}, F_{1}^{\prime}, p\right)$ (dashed line).

and (3) is therefore satisfied if and only if the following holds:

$$
\left(\frac{1-p_{\alpha}}{1-p_{\beta}}\right)^{k-1} \geq 1+\frac{\beta}{\alpha}
$$

Both sides of (4) are positive and greater than one. Thus, by taking logs on the left and right side, it is easy to show that the integral condition in (1) is satisfied if and only if the integration order $\widetilde{k}^{*}$ is large enough to verify:

$$
\widetilde{k}^{*} \geq 1+\frac{\ln (1+\beta / \alpha)}{\ln \left(1-p_{\alpha}\right)-\ln \left(1-p_{\beta}\right)} .
$$

Note that $\widetilde{k}^{*}$ is positive and greater than one and it always exists finite for any $0<$ $p_{\alpha}<p_{\beta}<1$ and for $\alpha, \beta>0$. Therefore the value $k^{*}$ exists as well, which concludes the proof.

\section{A.2 Proof of Proposition 1}

Proposition 1 EZOP is satisfied on the set of preferences $\mathcal{R} \Rightarrow \forall p \in[0,1],\left|\Gamma\left(F_{1}, F_{1}^{\prime}, p\right)\right| \leq$ $\left|\Gamma\left(F_{0}, F_{0}^{\prime}, p\right)\right|$.

Proof. By contradiction.

Assume $\exists \tilde{p} \in] 0,1\left[\right.$ such that $\left|\Gamma\left(F_{1}, F_{1}^{\prime}, \tilde{p}\right)\right|>\left|\Gamma\left(F_{0}, F_{0}^{\prime}, \tilde{p}\right)\right|$. For $\pi \in\{0,1\}, \Gamma\left(F_{\pi}, F_{\pi}^{\prime}, p\right)$ is left continuous since $F_{\pi}$ and $F_{\pi}^{\prime}$ are left continuous. Hence, $\exists \epsilon>0$ such that $\forall p \in$ $[\tilde{p}-2 \epsilon, \tilde{p}],\left|\Gamma\left(F_{1}, F_{1}^{\prime}, p\right)\right|>\left|\Gamma\left(F_{0}, F_{0}^{\prime}, p\right)\right|$ and $\operatorname{sign}\left(\Gamma\left(F_{\pi}, F_{\pi}^{\prime}, p\right)\right)=\operatorname{sign}\left(\Gamma\left(F_{\pi}, F_{\pi}^{\prime}, \tilde{p}\right)\right)$.

Consider the individual preferences $\tilde{W}$ given by the triangular weighting scheme over the interval $[\tilde{p}-2 \epsilon, \tilde{p}]: \forall p \in[0,1], \tilde{w}(p)=\left[(\epsilon-|p-(\tilde{p}-\epsilon)|) / \epsilon^{2}\right] \cdot \mathbb{1}_{p \in[\tilde{p}-2 \epsilon, \tilde{p}]}$, where $\mathbb{1}$ denotes the indicator function. For preferences $\tilde{W}$, the economic distance in social state $\pi$ is given by: $\left|\Delta_{\tilde{W}}\left(F, F^{\prime}\right)\right|=\int_{\tilde{p}-2 \epsilon}^{\tilde{p}} \tilde{w}(p)\left|\Gamma\left(F, F^{\prime}, p\right)\right| d p$. Henceforth $\left|\Delta_{\tilde{W}}\right|\left(F_{1}, F_{1}^{\prime}\right)|>| \Delta_{\tilde{W}}\left(F_{0}, F_{0}^{\prime}\right) \mid$ which violates equalization.

The reciprocal is not true. Figure 2 provides a counter-example. The plain line gives the gap curve under $\pi=0$. At value $p^{*}$, the curve crosses the horizontal axis. Hence, under $\pi=0$, type $c$ receives higher outcomes than type $c^{\prime}$ in the bottom of the distribution but lower outcomes in the top. Define the areas $A=\int_{0}^{p^{*}} \Gamma\left(F_{0}, F_{0}^{\prime}, p\right) d p$ and $B=-\int_{p^{*}}^{1} \Gamma\left(F_{0}, F_{0}^{\prime}, p\right) d p>0$. Now consider the weighting scheme that gives weight $\alpha \geq 0$ 
for percentiles below $p^{*}$ and $\beta \geq 0$ above $3^{3}$ The economic distance for this weighting scheme is $|\alpha A-\beta B|$. For $\alpha$ close enough to zero, type $c^{\prime}$ is preferred to type $c$ and the distance is given by $\beta B-\alpha A$. Under $\pi=1$, the gap curve is given by the dashed line, which is similar to the solid line except that the advantage of type $c$ has been reduced by a small cumulative amount $\epsilon$ in the bottom part of the distribution, so that $\int_{0}^{p^{*}} \Gamma\left(F_{1}, F_{1}^{\prime}, p\right) d p=A-\epsilon<A$. Gap curve dominance is obviously satisfied 4 At the same time, individuals who initially preferred the distribution of type $c^{\prime}$ to that of type $c$ will agree that the economic distance between type $c$ and $c^{\prime}$ has increased and EZOP is thus violated.

\section{A.3 Proof of Proposition 2}

Proposition 2 If $\forall \pi F_{\pi} \succ_{I S D 1} F_{\pi}^{\prime}$ then: EZOP over the set of preferences $\mathcal{R} \Leftrightarrow \forall p \in$ $[0,1], \Gamma\left(F_{0}, F_{0}^{\prime}, p\right) \geq \Gamma\left(F_{1}, F_{1}^{\prime}, p\right)$.

Proof. For the sufficiency part, assume that $F_{0} \succcurlyeq_{I S D 1} F_{0}^{\prime}$ and $F_{1} \succcurlyeq_{I S D 1} F_{1}^{\prime}$. As a consequence:

$$
\forall W \in \mathcal{R}, \forall \pi \int_{0}^{1} w(p) F_{\pi}^{-1}(p) d p>\int_{0}^{1} w(p) F_{\pi}^{\prime-1}(p) d p .
$$

Consequently, for all $W \in \mathcal{R}$, we can write:

$$
\Delta_{W}\left(F_{\pi}, F_{\pi}^{\prime}\right)=\int_{0}^{1} w(p) \Gamma\left(F_{\pi}, F_{\pi}^{\prime}, p\right) d p
$$

Hence, we have:

$$
\Delta_{W}\left(F_{0}, F_{0}^{\prime}\right)-\Delta_{W}\left(F_{1}, F_{1}^{\prime}\right)=\int_{0}^{1} w(p)\left[\Gamma\left(F_{0}, F_{0}^{\prime}, p\right)-\Gamma\left(F_{1}, F_{1}^{\prime}, p\right)\right] d p .
$$

If $\left[\Gamma\left(F_{0}, F_{0}^{\prime}, p\right)-\Gamma\left(F_{1}, F_{1}^{\prime}, p\right)\right] \geq 0$ for all $p$, since the weights $w(p)$ are non-negative, the integrand in equation (5) is positive for all $p$ and the integral is positive.

For the necessity part, note that if $\left[\Gamma\left(F_{0}, F_{0}^{\prime}, p\right)-\Gamma\left(F_{1}, F_{1}^{\prime}, p\right)\right]$ is negative in the neighborhood of a quantile $p_{0}$, we can find a weight profile $w(p)$ that is arbitrarily small outside this neighborhood and it makes the integral negative.

\section{A.4 Proof of Proposition 3}

Proposition 3 If $\forall \pi F_{\pi} \succ_{I S D 2} F_{\pi}^{\prime}$ then: EZOP over the set of preferences $\mathcal{R}^{2} \Leftrightarrow \forall p \in$ $[0,1], \int_{0}^{p} \Gamma\left(F_{0}, F_{0}^{\prime}, t\right) d t \geq \int_{0}^{p} \Gamma\left(F_{1}, F_{1}^{\prime}, t\right) d t$

Proof. As a consequence of the dominance hypothesis, we have:

$$
\forall W \in \mathcal{R}^{2}, \forall \pi \int_{0}^{1} w(p) F_{\pi}^{-1}(p) d p>\int_{0}^{1} w(p) F_{\pi}^{\prime-1}(p) d p .
$$

Consequently, for all $W \in \mathcal{R}^{2}$, we can write:

$$
\Delta_{W}\left(F_{\pi}, F_{\pi}^{\prime}\right)=\int_{0}^{1} w(p) \Gamma\left(F_{\pi}, F_{\pi}^{\prime}, p\right) d p .
$$

\footnotetext{
${ }^{3}$ This weighting scheme is given by $w(p)=\alpha+(\beta-\alpha) \cdot \mathbb{1}_{p>p^{*}}$, with $\alpha, \beta \geq 0$ and $\alpha p^{*}+\beta\left(1-p^{*}\right)=1$.

${ }^{4}$ The same result would obtain even in cases where $A<B$ and the weighting scheme is coherent with risk aversion, i.e. when $\alpha>\beta$.
} 
Hence, $\forall W \in \mathcal{R}^{2}$ we have:

$$
\Delta_{W}\left(F_{0}, F_{0}^{\prime}\right)-\Delta_{W}\left(F_{1}, F_{1}^{\prime}\right)=\int_{0}^{1} w(p)\left[\Gamma\left(F_{0}, F_{0}^{\prime}, p\right)-\Gamma\left(F_{1}, F_{1}^{\prime}, p\right)\right] d p .
$$

It is possible to integrate (6) by parts once,

$$
\begin{aligned}
\Delta_{W}\left(F_{0}, F_{0}^{\prime}\right)-\Delta_{W}\left(F_{1}, F_{1}^{\prime}\right)= & w(1) \int_{0}^{1}\left[\Gamma\left(F_{0}, F_{0}^{\prime}, p\right)-\Gamma\left(F_{1}, F_{1}^{\prime}, p\right)\right] d p \\
& +\int_{0}^{1}(-1) w^{\prime}(p) \int_{0}^{p}\left[\Gamma\left(F_{0}, F_{0}^{\prime}, t\right)-\Gamma\left(F_{1}, F_{1}^{\prime}, t\right)\right] d t d p
\end{aligned}
$$

By $W \in \mathcal{R}^{2}$ then $w(1)=0$ and the first term disappears. By $w^{\prime}(p) \leq 0$ for all $p$ makes $\int_{0}^{p}\left[\Gamma\left(F_{0}, F_{0}^{\prime}, t\right)-\Gamma\left(F_{1}, F_{1}^{\prime}, t\right)\right] d t \geq 0 \forall p \in[0,1]$ sufficient for $\Delta_{W}\left(F_{0}, F_{0}^{\prime}\right)-\Delta_{W}\left(F_{1}, F_{1}^{\prime}\right) \geq 0$ in (6), which gives the EZOP condition.

For the necessity part, the set of admissible evaluation functions is restricted to $W \in$ $\mathcal{R}^{2}$, which implies that $w(1)=0$ and $-w^{\prime}(p)$ is a bounded, continuous and non-negative function on $[0,1]$. Under regularity assumptions, $\int_{0}^{p}\left[\Gamma\left(F_{0}, F_{0}^{\prime}, t\right)-\Gamma\left(F_{1}, F_{1}^{\prime}, t\right)\right] d t$ must also be bounded and continuous on $[0,1]$. It then follows that it must be non-negative on $[0,1]$ and positive for at least one $p \in[0,1]$ (see Hadar and Russell 1969).

\section{A.5 Proof of Proposition 4}

Proposition 4 If $\forall \pi F_{\pi} \succ_{I S D \kappa} F_{\pi}^{\prime}$ then: EZOP over the set of preferences $\mathcal{R}^{\kappa} \Leftrightarrow \forall p \in$ $[0,1], \Gamma^{\kappa}\left(F_{0}, F_{0}^{\prime}, p\right) \geq \Gamma^{\kappa}\left(F_{1}, F_{1}^{\prime}, p\right)$.

Proof. As a consequence of the dominance hypothesis, we have:

$$
\forall W \in \mathcal{R}^{k}, \forall \pi \int_{0}^{1} w(p) F_{\pi}^{-1}(p) d p>\int_{0}^{1} w(p) F_{\pi}^{\prime-1}(p) d p .
$$

Consequently, for all $W \in \mathcal{R}^{k}$, we can write:

$$
\Delta_{W}\left(F_{\pi}, F_{\pi}^{\prime}\right)=\int_{0}^{1} w(p) \Gamma\left(F_{\pi}, F_{\pi}^{\prime}, p\right) d p .
$$

Hence, $\forall W \in \mathcal{R}^{k}$ we have:

$$
\Delta_{W}\left(F_{0}, F_{0}^{\prime}\right)-\Delta_{W}\left(F_{1}, F_{1}^{\prime}\right)=\int_{0}^{1} w(p)\left[\Gamma\left(F_{0}, F_{0}^{\prime}, p\right)-\Gamma\left(F_{1}, F_{1}^{\prime}, p\right)\right] d p .
$$

It is possible to integrate (7) by parts $k$ times,

$$
\begin{aligned}
\Delta_{W}\left(F_{0}, F_{0}^{\prime}\right)-\Delta_{W}\left(F_{1}, F_{1}^{\prime}\right)= & w(1) \int_{0}^{1}\left[\Gamma\left(F_{0}, F_{0}^{\prime}, p\right)-\Gamma\left(F_{1}, F_{1}^{\prime}, p\right)\right] d p \\
& +\sum_{j=1}^{k-1}(-1)^{j} \frac{d^{j} w(1)}{d p^{j}}\left[\Gamma^{k}\left(F_{0}, F_{0}^{\prime}, 1\right)-\Gamma^{k}\left(F_{1}, F_{1}^{\prime}, 1\right)\right] \\
& +(-1)^{k-1} \int_{0}^{1} \frac{d^{k-1} w(p)}{d p^{k-1}}\left[\Gamma^{k}\left(F_{0}, F_{0}^{\prime}, p\right)-\Gamma^{k}\left(F_{1}, F_{1}^{\prime}, p\right)\right] d p .
\end{aligned}
$$


By $W \in \mathcal{R}^{k}$ then $w(1)=0$ and $\frac{d^{j} w(1)}{d p^{j}}=0$ for all $j \leq k-1$ and the first and second terms above disappear. The fact that the sign of $(-1)^{j} \frac{d^{j} w(p)}{d p^{j}}$ is always positive for any $j$ by construction makes the conditions $\left[\Gamma^{k}\left(F_{0}, F_{0}^{\prime}, p\right)-\Gamma^{k}\left(F_{1}, F_{1}^{\prime}, p\right)\right] \geq 0 \forall p \in[0,1]$ sufficient for $\Delta_{W}\left(F_{0}, F_{0}^{\prime}\right)-\Delta_{W}\left(F_{1}, F_{1}^{\prime}\right) \geq 0$ as in (7), which defines the EZOP condition.

For the necessity part, the set of admissible evaluation functions is restricted to $W \in$ $\mathcal{R}^{k}$, which implies

$$
\Delta_{W}\left(F_{0}, F_{0}^{\prime}\right)-\Delta_{W}\left(F_{1}, F_{1}^{\prime}\right)=(-1)^{k-1} \int_{0}^{1} \frac{d^{k-1} w(p)}{d p^{k-1}}\left[\Gamma^{k}\left(F_{0}, F_{0}^{\prime}, p\right)-\Gamma^{k}\left(F_{1}, F_{1}^{\prime}, p\right)\right] d p
$$

with $(-1)^{k-2} \frac{d^{k-1} w(p)}{d p^{k-1}}$ bounded, continuous and non-negative function on [0, 1]. Under regularity assumptions, $\left[\Gamma^{k}\left(F_{0}, F_{0}^{\prime}, p\right)-\Gamma^{k}\left(F_{1}, F_{1}^{\prime}, p\right)\right]$ must also be bounded and continuous on $[0,1]$. It then follows that it must be non-negative on $[0,1]$ and positive for at least one $p \in[0,1]$ (see Hadar and Russell 1969).

\section{A.6 EZOP conditions with Von Neuman Morgenstern preferences}

Consider the setting of section 2 in the article, with a single effort level and circumstances $c$ and $c^{\prime}$ with CDF $F_{\pi}$ and $F_{\pi}^{\prime}$ respectively under social state $\pi$. Assume that $y$ has bounded support $[0, \bar{y}]$. Define $\mathcal{V}$ the set of VNM preferences. $W \in \mathcal{V}$ if and only if it can be expressed as :

$$
W(F)=\int_{0}^{\bar{y}} u(y) d F(y)
$$

with $u(y)>0$ and $u^{\prime}(y)>0$ for all $y \geq 0$. Redefine the gap curve on the basis of the CDF instead of the quantile functions : $\Gamma_{\pi}(y)=\Gamma\left(F_{\pi}, F_{\pi}^{\prime}, y\right)=F_{\pi}^{\prime}(y)-F_{\pi}(y)$.

The following proposition can be established :

Proposition 5 If $\forall \pi F_{\pi} \succ_{S D 1} F_{\pi}^{\prime}$ then: EZOP over the set of preferences $\mathcal{V} \Leftrightarrow \forall y \in$ $[0, \bar{y}], \Gamma_{0}(y) \geq \Gamma_{1}(y)$.

Proof. Using integration by parts, the welfare derived from the opportunity set $F$ can be expressed as:

$$
\begin{aligned}
W(F) & =[u(y) F(y)]_{0}^{\bar{y}}-\int_{0}^{\bar{y}} u^{\prime}(y) F(y) d y \\
& =u(\bar{y})-\int_{0}^{\bar{y}} u^{\prime}(y) F(y) d y
\end{aligned}
$$

Consequently, the absolute welfare gap between circumstances $c$ and $c^{\prime}$ under social state $\pi$ can be written as

$$
\Delta_{W}\left(F_{\pi}, F_{\pi}^{\prime}\right)=\left|-\int_{0}^{\bar{y}} u^{\prime}(y)\left[F_{\pi}(y)-F_{\pi}^{\prime}(y)\right] d y\right|
$$

If $\forall \pi F_{\pi} \succ_{S D 1} F_{\pi}^{\prime}$, the absolute welfare gap can be rexpressed as :

$$
\Delta_{W}\left(F_{\pi}, F_{\pi}^{\prime}\right)=\int_{0}^{\bar{y}} u^{\prime}(y)\left[F_{\pi}^{\prime}(y)-F_{\pi}(y)\right] d y
$$


Henceforth the change in unfair advantage can be written as :

$$
\begin{aligned}
\Delta_{W}\left(F_{0}, F_{0}^{\prime}\right)-\Delta_{W}\left(F_{1}, F_{1}^{\prime}\right) & =\int_{0}^{\bar{y}} u^{\prime}(y)\left\{\left[F_{0}^{\prime}(y)-F_{0}(y)\right]-\left[F_{1}^{\prime}(y)-F_{1}(y)\right]\right\} d y \\
& =\int_{0}^{\bar{y}} u^{\prime}(y)\left[\Gamma_{0}(y)-\Gamma_{1}(y)\right] d y,
\end{aligned}
$$

where $u()$ satisfies $u^{\prime}(y)>0 \forall y \in[0, \bar{y}]$. Hence $\Gamma_{0}(y) \geq \Gamma_{1}(y)$ implies $\int_{0}^{\bar{y}} u^{\prime}(y)\left[\Gamma_{0}(y)-\Gamma_{1}(y)\right] d y \geq$ 0 . Now assume that $\Gamma_{0}(y)-\Gamma_{1}(y)<0$ over some interval $[a, b]$ and $\Gamma_{0}(y)-\Gamma_{1}(y) \geq 0$ elsewhere. Consider the function $u()$ such that $u^{\prime}(y)=1$ over $[a, b]$ and $u^{\prime}(y)=\varepsilon>0$ elsewhere. Noting that $\Gamma_{0}(y)-\Gamma_{1}(y) \leq F_{0}(y)$ for $y \notin[a, b]$, we have :

$$
\begin{aligned}
\Delta_{W}\left(F_{0}, F_{0}^{\prime}\right)-\Delta_{W}\left(F_{1}, F_{1}^{\prime}\right)= & \int_{0}^{a} u^{\prime}(y)\left[\Gamma_{0}(y)-\Gamma_{1}(y)\right] d y+\int_{a}^{b} u^{\prime}(y)\left[\Gamma_{0}(y)-\Gamma_{1}(y)\right] d y+ \\
& +\int_{b}^{\bar{y}} u^{\prime}(y)\left[\Gamma_{0}(y)-\Gamma_{1}(y)\right] d y \\
\leq & \varepsilon\left[F_{0}(a)+1-F_{0}(b)\right]+\int_{a}^{b}\left[\Gamma_{0}(y)-\Gamma_{1}(y)\right] d y
\end{aligned}
$$

The term on the right hand side in the second line is negative for $\varepsilon$ sufficiently small. Hence if $\Gamma_{0}(y)-\Gamma_{1}(y)<0$ over some closed interval, there exists a function $u \in \mathcal{V}$ such that EZOP is violated.

If types' distributions cannot be ordered by SD1 in at least one state, then consensus has to be reduced to the class of evaluation functions displaying the VNM representation and that are increasing in income and display risk-aversion. The latter feature implies that EZOP has to be tested within the class of VNM preferences where $u^{\prime}(y)>0$ and $u^{\prime \prime}(y)<0$ for any $y \geq 0$, which is denoted $\mathcal{V}^{2} \subset \mathcal{V}$. A natural question is whether EZOP can be identified in this context. To obtain results, assume first that within each state $\pi$ types $c$ and $c^{\prime}$ are ordered by stochastic dominance at order 2, denoted $F_{\pi} \succ_{S D 2} F_{\pi}^{\prime}$ and meaning that all risk-averse preferences in $\mathcal{V}^{2}$ would agree that the the opportunity profile $F_{\pi}$ is preferred to $F_{\pi}^{\prime}$.

Testing SD2 is possible by checking if $S_{\pi}(y) \leq S_{\pi}^{\prime}(y), y \geq 0$, where $S_{\pi}(y)$ is the supercumulative distribution function of $F_{\pi}(y)$, denoted $S(y)=\int_{0}^{y} F(x) d x$. We can further develop on this notation to derive second order gap curves for distributions $F_{\pi}^{\prime}$ and $F_{\pi}$, denoted $\Gamma_{\pi}^{2}(y)=S_{\pi}^{\prime}(y)-S_{\pi}(y)$ for any $y \geq 0$. We now study the relation between integrated gap curves and welfare gaps $\Delta_{W}\left(F_{\pi}, F_{\pi}^{\prime}\right)=\left|W\left(F_{\pi}\right)-W\left(F_{\pi}^{\prime}\right)\right|$ with $W \in \mathcal{V}^{2}$. To do so, we further assume that the domain of incomes is bounded above at $\bar{y}$, so that $y \in[0, \bar{y}]$.

Integrating expected utility by parts twice yields:

$$
W(F)=u(\bar{y})-u^{\prime}(\bar{y}) S(\bar{y})+\int_{0}^{\bar{y}} u^{\prime \prime}(y) S(y) d y .
$$

Hence the welfare gap is given by :

$$
\begin{aligned}
\Delta_{W}\left(F_{\pi}^{\prime}, F_{\pi}\right) & =\left|-u^{\prime}(\bar{y})\left[S_{\pi}(\bar{y})-S_{\pi}^{\prime}(\bar{y})\right]+\int_{0}^{\bar{y}} u^{\prime \prime}(y)\left[S_{\pi}(y)-S_{\pi}^{\prime}(y)\right] d y\right| \\
& =\left|u^{\prime}(\bar{y}) \Gamma_{\pi}^{2}(\bar{y})-\int_{0}^{\bar{y}} u^{\prime \prime}(y) \Gamma_{\pi}^{2}(y) d y\right| .
\end{aligned}
$$


Define $\mu_{\pi}=\int_{0}^{\bar{y}} y d F_{\pi}(y)$ the mean of distribution of $F_{\pi}()$. This gives

$$
\begin{aligned}
S_{\pi}(\bar{y}) & =\int_{0}^{\bar{y}} F(y) d y \\
& =[y F(y)]_{0}^{\bar{y}}-\int_{0}^{\bar{y}} F(y) d y \\
& =\bar{y}-\mu_{\pi},
\end{aligned}
$$

from which follows $\Gamma_{\pi}^{2}(\bar{y})=\mu_{\pi}-\mu_{\pi}^{\prime}$. Instead of working with $\Delta_{W}\left(F_{\pi}, F_{\pi}^{\prime}\right)$ we can assess EZOP on the basis of $\tilde{\Delta}_{W}\left(F_{\pi}, F_{\pi}^{\prime}\right)=\Delta_{W}\left(F_{\pi}, F_{\pi}^{\prime}\right) / u^{\prime}(\bar{y})$ which is a linear transformation. We have :

$$
\tilde{\Delta}_{W}\left(F_{\pi}, F_{\pi}^{\prime}\right)=\left|\left[\mu_{\pi}-\mu_{\pi}^{\prime}\right]-\frac{1}{u^{\prime}(\bar{y})} \int_{0}^{\bar{y}} u^{\prime \prime}(y) \Gamma_{\pi}^{2}(y) d y\right|
$$

If $F_{\pi} \succ_{S D 2} F_{\pi}^{\prime}$ under both social states, we can get rid of absolute values ad have:

$$
\tilde{\Delta}_{W}\left(F_{\pi}, F_{\pi}^{\prime}\right)=\left[\mu_{\pi}-\mu_{\pi}^{\prime}\right]-\frac{1}{u^{\prime}(\bar{y})} \int_{0}^{\bar{y}} u^{\prime \prime}(y) \Gamma_{\pi}^{2}(y) d y \geq 0 .
$$

Robust equalization of opportunity within the class of preferences $\mathcal{V}^{2}$ is defined in this framework by $\tilde{\Delta}_{W}\left(F_{0}, F_{0}^{\prime}\right)-\tilde{\Delta}_{W}\left(F_{1}, F_{1}^{\prime}\right)>0$ for all $W \in \mathcal{V}^{2}$. It follows from $(8)$ that this condition requires to asses evolution of integrated gap curves, measuring the change in unfair income gaps across types, as well as the evolution of the average gaps.

When the change in the social state is such that the average economic gaps across types remain constant $\left(\mu_{0}-\mu_{0}^{\prime}=\mu_{1}-\mu_{1}^{\prime}\right)$, then gap curve dominance, i.e., $\Gamma_{0}^{2}(y) \geq \Gamma_{1}^{2}(y)$ for every $y \in[0, \bar{y}]$, defines a necessary and sufficient condition for EZOP within the class of evaluation functions $\mathcal{V}^{2}$.

When there is convergence in means across types $\left(\mu_{0}-\mu_{0}^{\prime}>\mu_{1}-\mu_{1}^{\prime}\right)$, then gap curve dominance, $\Gamma_{0}^{2}(y) \geq \Gamma_{1}^{2}(y)$ for every $y \in[0, \bar{y}]$, defines only a sufficient condition for EZOP. In fact, in this case the effect of a change in policy that induces convergence in the average value of income profiles can overcompensate for the loss in welfare generated by lack of gap dominance.

Finally, when there is divergence in the means $\left(\mu_{0}-\mu_{0}^{\prime}<\mu_{1}-\mu_{1}^{\prime}\right)$ then gap curve dominance of $\pi=0$ over $\pi=1$, i.e., $\Gamma_{0}^{2}(y) \leq \Gamma_{1}^{2}(y)$ for every $y \in[0, \bar{y}]$, can be used to test the presence of a robust disequalization of opportunity.

\section{B Anonymous EZOP between multiple types}

The definition of EZOP between multiple types (definition 2 in the article), makes the "identity" of each type relevant for defining equalization of opportunity. According to the definition, the extent of advantage between any pair of types $\left(c_{i}, c_{j}\right)$ under $\pi=0$ has to be confronted with the extent of advantage between the same two types under $\pi=1$. One may challenge this view and claim that only the magnitude of the gaps (and not the identity of the types involved) is relevant for defining equalization of opportunity. Consider a simple example in which there are three types $c_{1}, c_{2}$ and $c_{3}$. Assume that there is only one effort level and luck plays no role. Under each of the three states $\pi=A, B, C$, each type is assigned with an outcome given by the following table: 


\begin{tabular}{lccc}
\hline \hline & \multicolumn{3}{c}{ Outcomes } \\
\hline & $\pi=A$ & $\pi=B$ & $\pi=C$ \\
\cline { 2 - 5 }$c_{1}$ & 6 & 6 & 6 \\
$c_{2}$ & 3 & 4 & 2.5 \\
$c_{3}$ & 1 & 2.5 & 4 \\
\hline \hline
\end{tabular}

When moving from state $A$ to $B$, the gap between each type and the other two falls (the gap between $c_{1}$ and $c_{2}$ shrinks from $6-3$ to $6-4$ ) and the condition in definition 2 is satisfied. On the contrary, when moving from state $A$ to $C$, the gap between $c_{1}$ and $c_{2}$ increases and definition 2 is not satisfied, although state $C$ is obtained from state $B$ only by permuting the outcomes of groups $c_{2}$ and $c_{3}$. Arguably, situations $B$ and $C$ can be regarded to as equivalent: equality of opportunity is not satisfied in both cases and the unfair gaps generating inequality of opportunity have exactly the same magnitude in both situations, although they are associated to different types (that are a-priori not ordered). There are hence reasons to challenge the idea in definition 2 that equalization of opportunity statement should be sensitive to the identity of the groups generating a given opportunity gap.

The example above echoes the need for an anonymity principle, which is generally accepted in the assessment of inequality. According to this principle, the measurement of inequality of outcome should be insensitive to a permutation of the outcomes of individuals within the distribution. This principle can be incorporated in our definition of equalization of opportunity by making it insensitive to a permutation of the opportunity sets across types when moving from a social state to another.

Let us introduce some additional notation. Let $r_{\pi}^{W}(c)$ be the rank function assigning to circumstance $c$ its rank in the ranking of types, in social state $\pi$, according to preferences $W$. Given $W$, all circumstances can be ranked, but the rank of a specific circumstance $c$ might change across social states and differs across preferences.

The anonymity principle of equalization of opportunity between multiple types shifts the focus of EZOP from the label of the types to the rank they occupy. The principle requires that the opportunity gap between two types sitting at given ranks falls when moving from social state 0 to 1 . This should hold for every pair of ranks and every utility function in $\mathcal{P}$.

Definition 1 (Anonymous EZOP between multiple types) Moving from state $\pi=$ 0 to $\pi=1$ equalizes opportunity over the set of circumstances $C$, at effort $e$, on the set of preferences $\mathcal{P}$, if and only if, for all preferences $W \in \mathcal{P}$, for all $(i, j, h, \ell) \in\{1, \ldots, T\}^{4}$ such that $r_{0}^{W}\left(c_{i}\right)=r_{1}^{W}\left(c_{h}\right)$ and $r_{0}^{W}\left(c_{j}\right)=r_{1}^{W}\left(c_{\ell}\right)$ we have: $\left|\Delta_{W}\left(F_{0}\left(. \mid c_{i}, e\right), F_{0}\left(. \mid c_{j}, e\right)\right)\right| \geq\left|\Delta_{W}\left(F_{1}\left(. \mid c_{h}, e\right), F_{1}\left(. \mid c_{\ell}, e\right)\right)\right|$.

Implementation of anonymous equalization requires first to define a class of preferences where agreement is reached on the rank of types according to the advantage they confer, and, second, to check if the advantage between circumstances occupying a similar rank is reduced when changing state from $\pi=0$ to $\pi=1$. Within the class $\mathcal{R}$, agreement on this ranking of circumstances is reached only for the evaluation functions in the intersection of all the sets $\mathcal{R}^{\kappa_{i j}}$, for all pairs $(i, j)$. The intersection is denoted by $\mathcal{R}^{\kappa_{\text {max }}}$, where $\kappa_{\max }=\max _{i, j \in\{1, \ldots, T\}}\left\{\kappa_{i j}\right\}$. Once this set is identified, gap curve dominance can be tested. It only provides, however, a necessary condition for agreement over $\mathcal{R}$. It become also sufficient in the special case when $\kappa_{\max }=1$. 
Proposition 6 EZOP between multiple types over the set of preferences $\mathcal{R} \Rightarrow \forall p \in[0,1]$, $\forall(i, j, h, \ell) \in\{1, \ldots, T\}^{4}$ such that $r_{0}^{W}\left(c_{i}\right)=r_{1}^{W}\left(c_{h}\right)$ and $r_{0}^{W}\left(c_{j}\right)=r_{1}^{W}\left(c_{\ell}\right)$, $\left|\Gamma^{\kappa_{\max }}\left(F_{0}\left(. \mid c_{i}, e\right), F_{0}\left(. \mid c_{j}, e\right), p\right)\right| \geq\left|\Gamma^{\kappa_{\max }}\left(F_{1}\left(. \mid c_{h}, e\right), F_{1}\left(. \mid c_{\ell}, e\right), p\right)\right|$.

The proof is based on the same arguments used in the proof of proposition 4 in the article. Proposition 6 requires that unfair advantage fall for all possible pairs of circumstances. This criterion remains disaggregated and demanding: It requires performing a large number of comparisons between pairs of types that must all be validated by all preferences in a sufficiently heterogeneous class.

However, some unfair gaps might be considered more worth compensating (i.e., one expect a large equalizing impact from the change in social state) than others, from a normative standpoint. For instance, the maxmin criterion of Roemer et al. (2003) amounts to assigning priority to people with circumstances at the bottom of the distribution of types 5 In definition 1 , this would correspond to restricting the scope of inter-type comparisons to pairs involving the bottom type. One alternative criteria, in line with the approach by Peragine (2004), consists instead to evaluate equalization through the evolution of between-types inequality. Even in this case, a necessary condition for dominance is that the situation of the worse-off type improves by effect of the policy. Other normative perspectives about opportunity equalization might endorse other types as reference. For this reason, we always refer to the reference distribution.

We now introduce a criterion for anonymous EZOP between multiple types that is conditional on a reference distribution. Equalization of opportunity occurs if and only if the welfare gap between each and every distribution associated to ordered types and the reference distribution in situation $\pi=0$ is larger than the gap between the distribution of the type in the corresponding raking and the reference distribution in situation $\pi=1$. We maintain anonymity of evaluation, which implies that all types, and the reference distribution, have to be ranked unanimously by all preferences $W \in \mathcal{P}$. We denote the reference distribution as $F_{\pi}^{R}($.$) , to remark that the reference distribution might change$ across configurations.

Definition 2 (Anonymous and conditional EZOP between multiple types) Moving from state $\pi=0$ to $\pi=1$ equalizes opportunity over the set of circumstances $C$, at effort $e$, on the set of preferences $\mathcal{P}$, if and only if, for all preferences $W \in \mathcal{P}$, for all $(i, h) \in\{1, \ldots, T\}^{2}$ such that $r_{0}^{W}\left(c_{i}\right)=r_{1}^{W}\left(c_{h}\right)$ we have: $\left|\Delta_{W}\left(F_{0}\left(. \mid c_{i}, e\right), F_{0}^{R}(. \mid e)\right)\right| \geq\left|\Delta_{W}\left(F_{1}\left(. \mid c_{h}, e\right), F_{1}^{R}(. \mid e)\right)\right|$.

Compared to Definition 1, in Definition 2 we restrict assessments of equalization to all comparisons involving each type opportunity set and the reference distribution. Consider the case where the reference distribution is given by the opportunity set of the dominated type in each state $\pi$. Let these types be $j, \ell \in\{1, \ldots, T\}^{2}$, then if $r_{0}^{W}\left(c_{j}\right)=r_{1}^{W}\left(c_{\ell}\right)=1$ for given $e$ and for every $W \in \mathcal{P}$ (i.e. $j$ and $\ell$ are the most disadvantaged types), then it is sufficient to substitute $F_{0}^{R}()=.F_{0}\left(. \mid c_{j}, e\right)$ and $F_{1}^{R}(. \mid e)=F_{1}\left(. \mid c_{\ell}, e\right)$ in Definition 2 to obtain the desired opportunity equalization test.

The anonymous and conditional EZOP criterion can be implemented upon the choice of the reference class of evaluation functions. There must be agreement within the class in raking all circumstances along with the reference distribution. Within the set $\mathcal{R}$, agreement on this ranking of circumstances is reached only for the evaluation functions in the

\footnotetext{
${ }^{5}$ For alternative criteria, see Roemer (2012).
} 
intersection of all the sets $\mathcal{R}^{\kappa_{i j}}$, for all pairs $(i, j)$. We denote this set $\mathcal{R}^{\kappa_{\text {max }}}$ as before. All pairs of distributions can be ranked for preferences in the set $\mathcal{R}^{k^{\max }}$. The reference distribution should also be included in the ranking. Once this set is identified, gap curve dominance can be tested. It only provides, however, a necessary condition for agreement over $\mathcal{R}$ as for Proposition 6 .

Proposition 7 EZOP between multiple types over the set of preferences $\mathcal{R} \Rightarrow \forall p \in[0,1]$, $\forall(i, h) \in\{1, \ldots, T\}^{2}$ such that $r_{0}^{W}\left(c_{i}\right)=r_{1}^{W}\left(c_{h}\right)$, $\left|\Gamma^{\kappa_{\max }}\left(F_{0}\left(. \mid c_{i}, e\right), F_{0}^{R}(. \mid e), p\right)\right| \geq\left|\Gamma^{\kappa_{\max }}\left(F_{1}\left(. \mid c_{h}, e\right), F_{1}^{R}(. \mid e), p\right)\right|$.

The necessary condition is implemented from choosing the reference distribution. In the application to kindergarten expansion, we apply proposition 7 to test the extent at which the gap between the least advantaged group with respect to other circumstances closes up. The test being rejected implies that equalization cannot be accepted.

\section{Statistical inference for gap curve dominance}

\section{C.1 Setting and null hypothesis}

Consider a sample $y_{1}, y_{2}, \ldots, y_{n}$ of $n$ draws from a random variable $Y$ with distribution $F$. Let assume for simplicity that $y_{1} \leq y_{2} \leq \ldots \leq y_{n}$, so that $y_{i}$ refers to the observation in position $i$ in the ranking. The empirical distribution for the sample is denoted $\widehat{F}(y)=$ $\frac{1}{n} \sum_{i=1}^{n} \mathbf{1}\left(y_{i} \leq y\right)$ while the empirical quantile function is denoted $\widehat{F}^{-1}(p)=\inf \{y: \widehat{F}(y) \geq$ $p$ \}. If $\widehat{F}$ is a consistent estimator for $F$, then $\widehat{\Lambda}^{k}$ is a consistent estimator for $\Lambda^{k}$.

The empirical counterparts of the distributions $F_{1}$ and $F_{1}^{\prime}$, corresponding to circumstances $c$ and $c^{\prime}$, are denoted $\widehat{F}_{1}$ and $\widehat{F}^{\prime}{ }_{1}$ respectively, where in general $n_{c, 1} \neq n_{c^{\prime}, 1}$. These distributions can be obtained from random samples drawn from a well defined population. In the policy evaluation case, in particular, the actual distributions are estimated from the sample of treated units (i.e., the sample of children born in post reform cohorts in treatment municipalities). In many cases, also $\widehat{F}_{0}$ and ${\widehat{F^{\prime}}}_{0}$ can be obtained from well defined random samples. In these situations, ISD $k$ inference procedures can be adopted to determine the dominating distribution ${ }^{6}$ In the policy evaluation setting, however, $\pi=0$ represents a counterfactual distribution that is only identified from the actual distribution and the policy effects. The two distributions $\widehat{F}_{0}$ and $\widehat{F}_{0}^{\prime}$ can be estimated by applying to $\widehat{F}_{1}^{-1}(p)$ and to ${\widehat{F^{\prime}}}_{1}^{-1}(p)$ the corresponding conditional QTE estimates $Q \hat{T} E(p \mid c)$ and $Q \hat{T} E\left(p \mid c^{\prime}\right)$ at every $p \in[0,1]$. In this case, inference procedures can be based on bootstrapped covariances and standard errors.

Whenever $F_{\pi} \succ_{I S D k} F_{\pi}^{\prime}$ for all $\pi$, the gap curves differences are well defined and gap curves dominance and equality null hypothesis can be stated by setting conditions on $\Lambda_{\pi}^{k}(p)$

\footnotetext{
${ }^{6}$ See Beach and Davidson (1983) and Zheng (2002) for estimators of quantile functions and generalized Lorenz functions coordinates, while see Andreoli (2017) for extensions of inference on ISD at orders higher than the second. See also Davidson and Duclos (2000) for inference procedures of direct stochastic dominance analysis.
} 
and $\Lambda_{\pi}^{\prime k}(p)$ in every state $\left.\pi\right]^{7}$

$$
\begin{array}{ll}
H_{0}^{k}: & \Lambda_{0}^{k}(p)-\Lambda_{0}^{\prime k}(p) \geq \Lambda_{1}^{k}(p)-\Lambda_{1}^{\prime k}(p) \quad \text { for all } p \in[0,1] \\
H_{1}^{k}: & \Lambda_{0}^{k}(p)-\Lambda_{0}^{\prime k}(p)<\Lambda_{1}^{k}(p)-\Lambda_{1}^{\prime k}(p) \quad \text { for some } p \in[0,1] .
\end{array}
$$

The random process $\Lambda^{k}(p)$ is, in general, continuous. However, it is more convenient to express equality and dominance null hypothesis as linear equality and inequality constraints on a finite number $m$ of abscissae $p \in\left\{p_{1}, \ldots, p_{m}\right\}$. The estimates of $\widehat{\Lambda}^{k}(p)$ corresponding to these abscissae are organized in a column vector of coordinates:

$$
\widehat{\Lambda}^{k}=\left(\widehat{\Lambda}^{k}\left(p_{1}\right), \ldots, \widehat{\Lambda}^{k}\left(p_{m}\right)\right)^{t}
$$

with $\boldsymbol{\Lambda}^{k}$ being the corresponding vector in the population. Within the discrete setting it can be shown that (see Andreoli 2017):

$$
\widehat{\boldsymbol{\Lambda}}^{k} \text { is asymptotically distributed as } \mathcal{N}\left(\boldsymbol{\Lambda}^{k}, \frac{\boldsymbol{\Sigma}^{k}}{n}\right),
$$

where we use $\frac{\widehat{\boldsymbol{\Sigma}}^{k}}{n}$ as the estimator of the asymptotic $m \times m$ covariance matrix of $\widehat{\boldsymbol{\Lambda}}^{k}$. As a consequence of asymptotic normality, test statistics for ISD $k$ and gap curve dominance relations have well known distributional properties. We retain asymptotic normality also for cases in which the covariances are bootstrapped.

\section{C.2 Application to Gap curves dominance}

We test dominance conditions for the gap curve by considering a finite number of thresholds where dominance has to be tested. The coordinates of the integrated quantile functions corresponding to these thresholds are summarized by vectors $\boldsymbol{\Lambda}_{\pi}^{k}$ and $\boldsymbol{\Lambda}_{\pi}^{\prime k}$, corresponding respectively to the population distributions $F_{\pi}$ and $F_{\pi}^{\prime}$ in both $\pi=0$ and $\pi=1$. We define $\boldsymbol{\Lambda}_{\Gamma}^{k}$ the $4 m \times 1$ vector obtained by staking the vectors $\boldsymbol{\Lambda}_{0}^{k}, \boldsymbol{\Lambda}_{0}^{\prime k}, \boldsymbol{\Lambda}_{1}^{k}$ and $\boldsymbol{\Lambda}_{1}^{\prime k}$ in this precise order. The corresponding estimates are collected in the $4 m \times 1$ vector $\widehat{\Lambda}_{\Gamma}^{k}$, and we use $n=n_{c, 0}+n_{c^{\prime}, 0}+n_{c, 1}+n_{c^{\prime}, 1}$ to denote the overall sample size, gathering together all observations in the sub-samples delimited by circumstances $c$ and $c^{\prime}$ under $\pi=0$ and $\pi=1$, while $r_{c, \pi}=n_{c, \pi} / n$ is the relative size of each sub-sample. In the case of bootstrapped estimators, as in the policy evaluation application, it is sufficient to set $n_{c, \pi}=1$.

The hypothesis of gap curve dominance can be reformulated as a sequence of $m$ linear constraints on the vector $\boldsymbol{\Lambda}_{\Gamma}^{k}$. Let $\mathbf{R}_{\Gamma}=(\mathbf{R},-\mathbf{R})$ be the $m \times 4 m$ difference-in-differences matrix, where $\mathbf{R}=(\mathbf{I},-\mathbf{I})$ and $\mathbf{I}$ is an identity matrix of size $m$. Define the parametric vector $\gamma_{k}=\mathbf{R}_{\Gamma} \boldsymbol{\Lambda}_{\Gamma}^{k}$.

We make two (non-testable) assumptions: (i) $F_{\pi}$ and $F_{\pi}^{\prime}$ are independent processes within any $\pi$; (ii) the independence extends also across policy regimes. This latter assumption is verified when the sampling scheme is based upon randomized assignment to

\footnotetext{
${ }^{7}$ We stress here an important difference between the null hypothesis for gap curve dominance $H_{0}^{k}$ and the hypotheses of dominance/equality formulated in stochastic dominance literature. In our setting, the null dominance/equality hypothesis is on the fact that the gap in distributions in policy state $\pi=0$ is larger/equal than/to the gap in distributions registered in policy state $\pi=1$. In stochastic dominance analysis, instead, the null hypothesis is generally formulated on the sign of this gap in each policy state separately. The goal of the two approaches is, therefore, different.
} 
treatment and control groups. Under the two assumptions of independence and using the result in (9), it holds that:

$$
\sqrt{n} \widehat{\boldsymbol{\gamma}}_{k}=\sqrt{n} \mathbf{R}_{\Gamma} \widehat{\boldsymbol{\Lambda}}_{\Gamma}^{k} \text { is asymptotically distributed as } \mathcal{N}\left(\sqrt{n} \mathbf{R}_{\Gamma} \boldsymbol{\Lambda}_{\Gamma}^{k}, \mathbf{\Phi}\right),
$$

where $\widehat{\gamma}_{k}$ denotes the sample estimate of $\gamma_{k}$, and

$$
\boldsymbol{\Phi}=\mathbf{R}_{\Gamma} \operatorname{diag}\left(\frac{\Sigma_{c, 0}^{k}}{r_{c, 0}}, \frac{\boldsymbol{\Sigma}_{c^{\prime}, 0}^{k}}{r_{c^{\prime}, 0}}, \frac{\boldsymbol{\Sigma}_{c, 1}^{k}}{r_{c, 1}}, \frac{\boldsymbol{\Sigma}_{c^{\prime}, 1}^{k}}{r_{c^{\prime}, 1}}\right) \mathbf{R}_{\Gamma}^{t} .
$$

The empirical estimator of the asymptotic variance, $\widehat{\boldsymbol{\Phi}}$, is obtained by plugging $\widehat{\boldsymbol{\Sigma}}_{c, \pi}^{k}$ in the previous formula. We now discuss, within this framework, the test statistics associated to equality and dominance null hypothesis for pairs of gap cures. We conclude the section by showing that the procedure can be simplified by using conditional QTE bootstrapped estimates, as proposed in the empirical session.

\section{C.3 Testing equality in gap curves}

The null and alternative hypothesis for equality in gap curves coordinates associated to the set of abscissae $\left\{p_{1}, \ldots, p_{m}\right\}$ are:

$$
H_{0}^{k}: \gamma_{k}=\mathbf{0} \quad H_{1}^{k}: \gamma_{k} \neq \mathbf{0}
$$

Under the null hypothesis, it is possible to resort to a Wald test static $T_{1}^{k}$ :

$$
T_{1}^{k}=n \widehat{\gamma}_{k}^{t} \widehat{\Phi}^{-1} \widehat{\gamma}_{k} .
$$

Given the convergence results in 10 , the asymptotic distribution of the test $T_{1}^{k}$ is $\chi_{m}^{2}$. The $p$-value tabulation follows the usual rules.

\section{C.4 Testing dominance in gap curves}

The null and alternative hypothesis for dominance in gap curves can be reformulated as a sequence of positivity constraints on the vector $\gamma_{k}$ :

$$
H_{0}^{k}: \gamma_{k} \in \mathbb{R}_{+}^{m} \quad H_{1}^{k}: \gamma_{k} \notin \mathbb{R}_{+}^{m}
$$

The Wald test statistics with inequality constraints has been developed by Kodde and Palm (1986). For this set of hypothesis, the test statistics $T_{2}^{k}$ is defined as:

$$
T_{2}^{k}=\min _{\gamma_{k} \in \mathbb{R}_{+}^{m}}\left\{n\left(\widehat{\gamma}_{k}-\gamma_{k}\right)^{t} \widehat{\boldsymbol{\Phi}}^{-1}\left(\widehat{\gamma}_{k}-\gamma_{k}\right)\right\}
$$

Kodde and Palm (1986) have shown that the statistic $T_{2}^{k}$ is asymptotically distributed as a mixture of $\chi^{2}$ distributions, provided that 10 holds:

$$
T_{2}^{k} \sim \bar{\chi}^{2}=\sum_{j=0}^{m} w(m, m-j, \widehat{\Phi}) \operatorname{Pr}\left(\chi_{j}^{2} \geq c\right),
$$


with $w(m, m-j, \widehat{\mathbf{\Phi}})$ the probability that $m-j$ elements of $\gamma_{k}$ are strictly positive 8

To test the reverse dominance order, that is $\Gamma\left(\Lambda_{1}^{k}, \Lambda_{1}^{\prime k}, p\right) \geq \Gamma\left(\Lambda_{0}^{k}, \Lambda_{0}^{\prime k}, p\right)$ for all $p \in$ $[0,1]$, it is sufficient to replace $-\widehat{\gamma}_{k}$ and $-\gamma_{k}$ for their positive counterparts.

\section{C.5 Testing equality and dominance using QTE}

When $F_{\pi} \succ_{I S D 1} F_{\pi}^{\prime}$ under all policy regimes, gap curves dominance can be assessed by comparing the full distribution of QTE associated to conditional distributions of the two types $c$ and $c^{\prime}$. In fact, in this case it can be established that:

$$
\Gamma\left(F_{0}, F_{0}^{\prime}, p\right)-\Gamma\left(F_{1}, F_{1}^{\prime}, p\right)=Q T E\left(p \mid c^{\prime}\right)-Q T E(p \mid c), \quad \forall p \in[0,1] .
$$

Gap curves dominance is equivalently assessed by checking if the QTE distribution associated to the type that is disadvantaged in every policy regime is larger than the corresponding QTE distribution of the advantaged type. This indicates that the policy gains of the disadvantaged type should outweight the policy gains attributable to the advantaged type. Since this holds at every conditional quantile of the two types distributions, the disadvantaged type catches up the advantaged type and opportunities are equalized for this pair. The baseline econometric models provides estimates of the QTE at different quantiles of the conditional outcomes distributions, along with their covariances. In the application, these estimators are obtained through bootstrap methods.

Let denote the vector of conditional QTE on the conditional distribution of type $c$ and calculated for a finite number of thresholds $p \in\left\{p_{1}, \ldots, p_{m}\right\}$ as

$$
\hat{\boldsymbol{\beta}}_{c}^{\prime}=\left(Q \hat{T} E\left(p_{1} \mid c\right), \ldots, Q \hat{T} E\left(p_{m} \mid c\right)\right) .
$$

In analogy with the previous notation, let $\widehat{\boldsymbol{\beta}}$ be the vector obtained by staking the QTE distributions $\widehat{\boldsymbol{\beta}}_{c}$ and $\widehat{\boldsymbol{\beta}}_{c^{\prime}}$ associated to the pair of circumstances $c, c^{\prime}$. Using bootstrapped estimates, we can obtain the whole covariance structure of $\widehat{\boldsymbol{\beta}}$, denoted $\widehat{\boldsymbol{\Sigma}}_{Q T E}$. The $m$ variate vector of parameters of interest is denoted $\widehat{\gamma}_{Q T E}=\mathbf{R} \widehat{\boldsymbol{\beta}}$. Under the normality assumption, the covariance of $\widehat{\gamma}_{Q T E}$, which is denoted $\boldsymbol{\Phi}_{Q T E}$, is given by:

$$
\boldsymbol{\Phi}_{Q T E}=n \mathbf{R} \widehat{\Sigma}_{Q T E} \mathbf{R}^{t}
$$

One can further assume that the QTE estimates associated to different groups are independent, to obtain simplified covariance structures similar to what discussed in the previous subsections.

In this setting, the hypothesis of equality of QTE, as well as the hypothesis that the QTE distribution of the disadvantaged type dominates the QTE distribution of the advantaged types, can be tested as shown in the previous sections, using the test statistics $T_{1}$ and $T_{2}$. Acceptance or rejections of these tests allow to accept or reject gap curve dominance. When $F_{\pi}$ and $F_{\pi}^{\prime}$ are not ordered in the same way by $I S D 1$, this procedure does not implement a test for gap curves dominance.

\footnotetext{
${ }^{8}$ To estimate $w(m, m-j, \widehat{\mathbf{\Phi}})$, we draw 10,000 multivariate normal vectors with covariance matrix $\widehat{\mathbf{\Phi}}$, provided it is positive definite. Then, we compute the proportion of vectors with $m-j$ positive entries.
} 


\section{Empirical implementation}

This section develops an algorithm that allows to test whether the ex ante EZOP condition is satisfied. Assume that individual outcome and circumstances are observed for a representative sample of the population. The following algorithm operationalizes the ex-ante EZOP criterion. The algorithm proposes null hypothesis for conditional outcome distribution and for gap curves, focusing in particular on equality and dominance null hypothesis. The appropriate inference procedure, tests statistics and their distributions that must be used to test these null hypothesis are treated in appendix C. Valid alternatives to equality and dominance are described in Dardanoni and Forcina (1999).

The algorithm defines a procedure for comparing pairs of distributions made conditional on circumstances $c_{i}, c_{j} \in C$ with $i, j \in\{1, \ldots, T\}$, the set of population types. In the application, types are defined by intervals of parental resources bounded by deciles of the family labor income, with $T=10$. Hereby, the algorithm develops a procedure for assessing the most demanding non-anonymous ex-ante opportunity equalization criterion. It can be easily adapted to check equalization with anonymity, or with respect to some reference type. We denote with the scalar $\kappa_{i j}(\pi)$ the minimal degree of ISD at which the two distributions can be ranked in a given social state $\pi$. The algorithm establishes the steps that must be iterated to determine empirically $\kappa_{i j}(\pi)$. Inference on this scalar remains beyond the scope of this work.

Algorithm 1 (Implementable ex ante EZOP for two types) The following sequence of estimations and tests implements EZOP:

(i) $\forall c_{i} \in C, \forall \pi$, estimate $F_{\pi}^{-1}\left(p \mid c_{i}\right)$ and its integrals $\Lambda_{\pi}^{k}\left(p \mid c_{i}\right)$.

(ii) For each $\left(c_{i}, c_{j}, \pi\right)$ with $i, j \in\{1, \ldots, T\}$ compute $\kappa_{i j}(\pi)$ as follows:

(a) Consider $k \in \mathbb{N}_{++}$, with $k=1$ for the first iteration;

(b) Given $k$, define and test the following pair of null hypothesis:

$\left\{H_{0}: F_{\pi}\left(y \mid c_{i}\right) \succ_{I S D k} F_{\pi}\left(y \mid c_{j}\right)\right.$ vs. $\left.H_{a}: F_{\pi}\left(y \mid c_{i}\right) \nsucc_{I S D k} F_{\pi}\left(y \mid c_{j}\right)\right\}$

and

$\left\{H_{0}: F_{\pi}\left(y \mid c_{j}\right) \succ_{I S D k} F_{\pi}\left(y \mid c_{i}\right)\right.$ vs. $\left.H_{a}: F_{\pi}\left(y \mid c_{j}\right) \succ_{I S D k} F_{\pi}\left(y \mid c_{i}\right)\right\}$.

(c) Define $I_{k}=(a, b)$ the result of this pair of tests, where $a, b$ is equal to 1 if the null hypothesis is rejected and 0 otherwise, respectively for both null hypothesis.

(d) Compute $I_{k}$ :

- if $I_{k}=(0,0): \kappa_{i j}(\pi)=\infty$ - stop.

- if $I_{k}=(0,1)$ or if $I_{k}=(1,0): \kappa_{i j}(\pi)=k$ - stop.

- if $I_{k}=(1,1)$ : let $k=k+1$ and iterate from step (b).

(iii) Define $\kappa_{i j}:=\max _{\pi}\left\{\kappa_{i j}(0), \kappa_{i j}(1)\right\}$.

(iv) Verify gap curve dominance at order $\kappa_{i j}$, where $c$ and $c^{\prime}$ represent respectively the dominating and dominated distribution out of the pair $c_{i}, c_{j}$ :

$\left\{H_{0}: \Gamma\left(\Lambda_{0}^{\kappa_{i j}}(p \mid c), \Lambda_{0}^{\kappa_{i j}}\left(p \mid c^{\prime}\right)\right) \geq \Gamma\left(\Lambda_{1}^{\kappa_{i j}}(p \mid c), \Lambda_{1}^{\kappa_{i j}}(p \mid c)\right) \forall p \in[0,1]\right.$ and $H_{a}: H_{0}$ is false $\}$.

- If $\kappa=1$ :

- If $H_{0}$ accepted: EZOP is verified.

- If $H_{0}$ accepted with equality: neutrality is verified. 
- If $H_{0}$ rejected: inconclusive, EZOP is rejected.

- If $\kappa \geq 2$ :

- If $H_{0}$ accepted: Necessary conditions for equalization are satisfied.

- If $H_{0}$ accepted with equality: neutrality is verified.

- If $H_{0}$ rejected: inconclusive, EZOP is rejected.

When $k=1$, gap curve dominance can be inferred from comparisons of QTE. Provided that $F_{\pi}(y \mid c) \succcurlyeq_{I S D 1} F_{\pi}\left(y \mid c^{\prime}\right)$, then the gap curve dominance relation

$\Gamma\left(F_{0}(y \mid c), F_{0}\left(y \mid c^{\prime}\right), p\right)-\Gamma\left(F_{1}(y \mid c), F_{1}\left(y \mid c^{\prime}\right), p\right)=F_{1}^{-1}\left(p \mid c^{\prime}\right)-F_{0}^{-1}\left(p \mid c^{\prime}\right)-\left(F_{1}^{-1}(p \mid c)-F_{0}^{-1}(p \mid c)\right)$

is, by definition of the QTE on conditional types distribution, equivalent to verify that $Q T E\left(p \mid c^{\prime}\right) \geq Q T E(p \mid c)$ for every conditional quantile $p \in[0,1]$. This result allows to use more precise estimators of the gap curves, although the equivalence is valid exclusively in the case $k=1$.

\section{E Additional material for the empirical application}

\section{E.1 Support analysis for QTE estimation}

The data used in this paper have been presented in detail by Havnes and Mogstad (2011, 2015). We address the reader to these papers to gather explanations for: (i) the sample composition; (ii) the effects of the Kindergarten Act on child participation in early education and on the effect of the policy on the labor supply of the mothers; (iii) the similarity between treated and control groups in terms of the characteristics of the municipalities they are associated to; (iv) the validity of the DiD identification strategy; (v) the computation of QTE of the policy using RIF-DiD, OLS and change-in-changes estimators; (vi) the definition and distribution of family earnings, along with the effect of the policy on intergenerational earnings elasticity.

Figure 3 reports a non-parametric estimates of the joint distribution of child earnings and parental earnings. The high symmetry of the cumulative distribution function ensure that there is enough support for the estimation of QTE for high quantiles of earnings even for the groups coming from less advantaged backgrounds, when running RIF-DiD estimations. The support requirements are satisfied at the bottom of the children earnings distribution, where the density of parental earnings is sufficiently high for all groups, as confirmed by panel (a) of figure 4 for selected parental earnings decile groups. The issues related to lack of support at the top of the children earnings distributions are of marginal relevance. Panel (b) of figure 4 shows in fact that, although the share of group D10 (10th parental earnings decile) is proportionally higher than the one of groups D1 or of D5 for top percentiles of the children earnings distributions, still each of these two groups represents half of the group D10 share at that specific percentile. Furthermore, we run conditional QTE models, so the estimates of the parameters $\boldsymbol{\beta}_{3}^{t} \cdot \mathbf{x}$ in (4) for very high income thresholds are mostly associated to top parental earnings deciles groups.

\section{E.2 EZOP test: higher order comparisons for selected decile groups}

The tests reported in figure 2 in the main article show that equalization cannot be rejected for a large majority of the groups involved in the gap curve dominance comparisons. This 


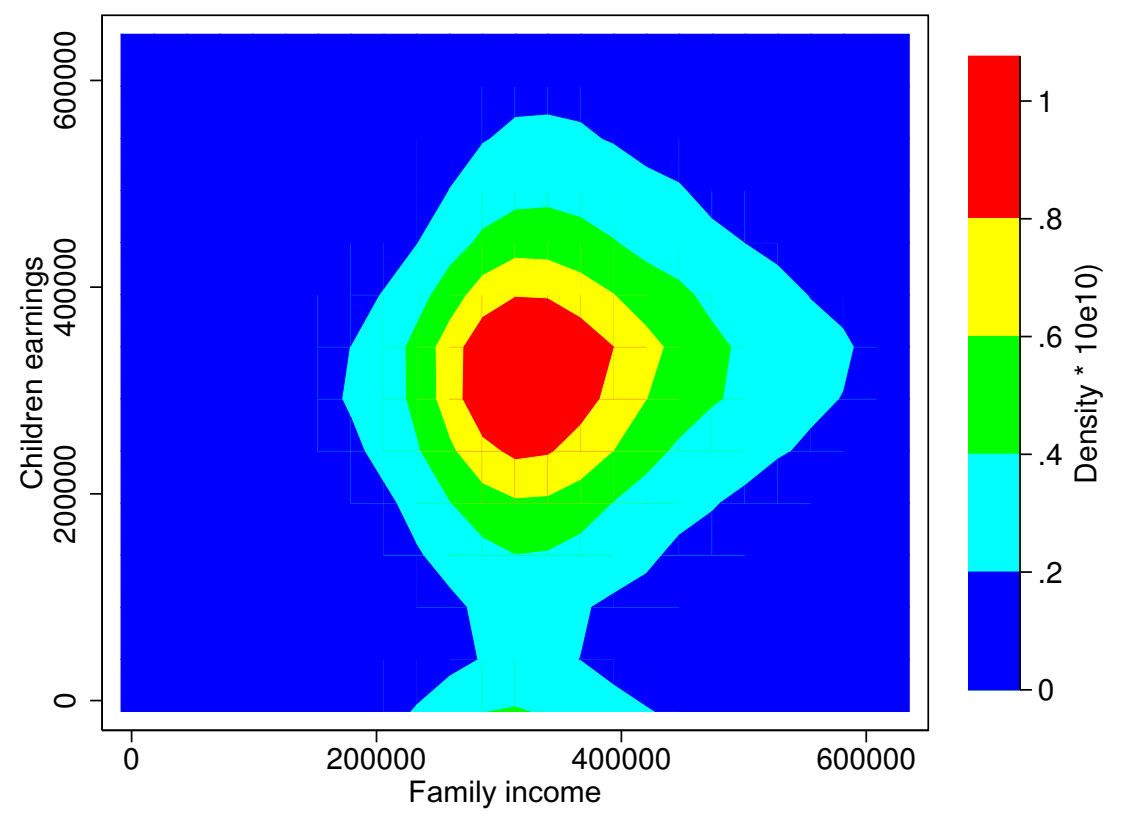

$\mathrm{t}$

Figure 3: Joint distribution of child earnings and parental earnings.

Note: Distribution is estimated using an epanechnikov kernel with Silverman ROT bandwidth, multiplied by $10 \mathrm{e} 10$ for notational simplicity.

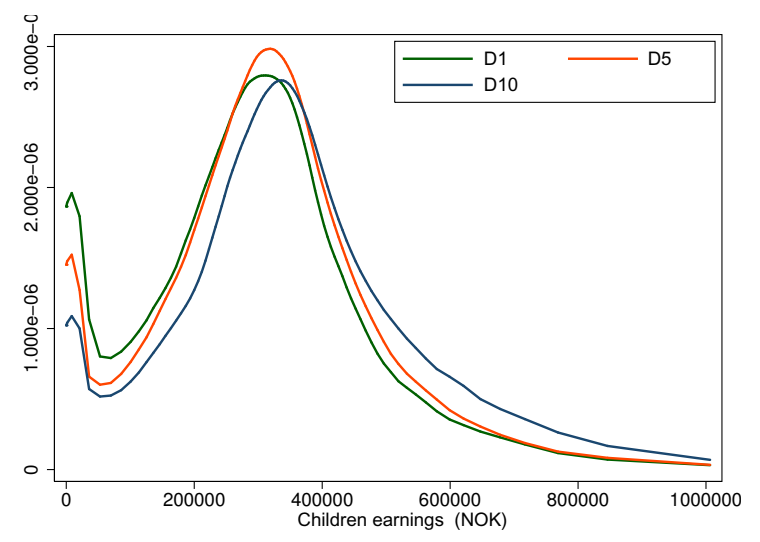

(a) Density of child earnings

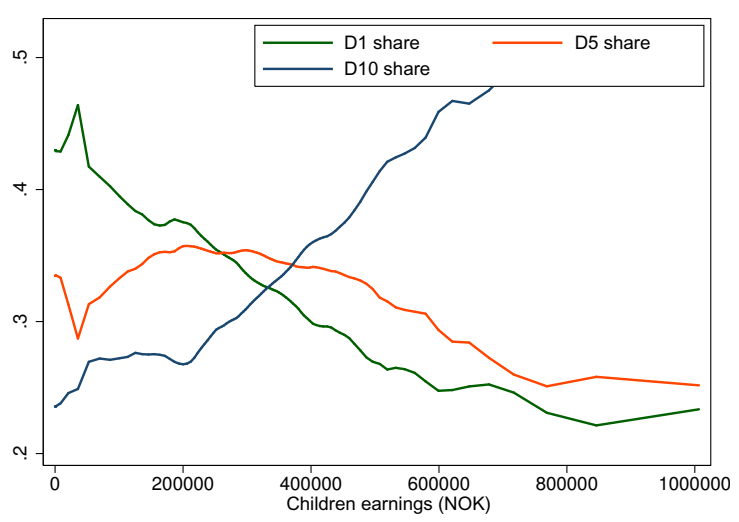

(b) Population shares by child earnings

Figure 4: Distribution of child earnings by selected parental earnings deciles groups.

Note: D1, D5 and D10 refer to children with parental earnings in the first, fifth, and tenth decile, respectively. Density estimates are calculated using an Epanechnikov kernel with Silverman ROT bandwidth. Panel (b) refers to shares in the population of children in these three deciles, estimated as the fraction of estimated kernel density to the sum of estimated kernel densities. 
Table 1: Equalization of opportunity for selected parental earnings deciles groups

\begin{tabular}{|c|c|c|c|c|c|c|c|}
\hline & \multirow{2}{*}{\multicolumn{6}{|c|}{$\begin{array}{l}\text { Pairwise groups comparisons: } \\
\text { D1 vs. }\end{array}$}} & \multirow{3}{*}{$\begin{array}{c}\text { D3 vs. } \\
\text { D5 }\end{array}$} \\
\hline & & & & & & & \\
\hline & $\mathrm{D} 4$ & D5 & D6 & D7 & D8 & D9 & \\
\hline \multicolumn{8}{|c|}{ A - Counterfactual generalized Lorenz, dominance relations $(\pi=0)$} \\
\hline \multirow[t]{2}{*}{$H_{0}: \sim$} & 118.9 & 155.8 & 280.4 & 423.9 & 702.0 & 851.7 & 44.4 \\
\hline & {$[0.000]$} & {$[0.000]$} & {$[0.000]$} & {$[0.000]$} & {$[0.000]$} & {$[0.000]$} & {$[0.001]$} \\
\hline \multirow[t]{2}{*}{$H_{0}: \succcurlyeq$} & 118.9 & 155.8 & 280.4 & 423.9 & 702.0 & 851.7 & 43.9 \\
\hline & {$[0.000]$} & {$[0.000]$} & {$[0.000]$} & {$[0.000]$} & {$[0.000]$} & {$[0.000]$} & {$[0.000]$} \\
\hline \multirow[t]{2}{*}{$H_{0}: \preccurlyeq$} & 0.0 & 0.0 & 0.0 & 0.0 & 0.0 & 0.0 & 4.4 \\
\hline & {$[0.769]$} & {$[0.781]$} & {$[0.778]$} & {$[0.776]$} & {$[0.782]$} & {$[0.781]$} & {$[0.600]$} \\
\hline \multicolumn{8}{|c|}{ B - Actual generalized Lorenz, dominance relations $(\pi=1)$} \\
\hline \multirow[t]{2}{*}{$H_{0}: \sim$} & 129.1 & 112.3 & 213.4 & 261.3 & 481.0 & 494.1 & 21.7 \\
\hline & {$[]$.} & {$[0.000]$} & {$[]$.} & {$[]$.} & {$[]$.} & {$[]$.} & {$[0.549]$} \\
\hline \multirow[t]{2}{*}{$H_{0}: \succcurlyeq$} & 129.1 & 112.3 & 213.4 & 261.3 & 481.0 & 494.1 & 21.7 \\
\hline & {$[]$.} & {$[0.000]$} & {$[]$.} & {$[]$.} & {$[]$.} & {$[]$.} & [0.318] \\
\hline \multirow[t]{2}{*}{$H_{0}: \preccurlyeq$} & 0.0 & 0.0 & 0.0 & 0.0 & 0.0 & 0.0 & 0.0 \\
\hline & {$[0.880]$} & {$[0.880]$} & {$[0.880]$} & {$[0.883]$} & {$[0.885]$} & {$[0.882]$} & [0.883] \\
\hline \multicolumn{8}{|c|}{ C - Gap curve equality and dominance tests at order ISD2 $(\pi=0$ vs $\pi=1)$} \\
\hline \multirow{2}{*}{$H_{0}:$ Neutrality } & 43.1 & 58.2 & 87.1 & 110.4 & 163.0 & 193.0 & 60.9 \\
\hline & [0.036] & {$[0.003]$} & {$[0.000]$} & {$[0.000]$} & {$[]$.} & [.] & [0.002] \\
\hline \multirow[t]{2}{*}{$H_{0}:$ Equalization } & 49.4 & 59.0 & 75.4 & 134.4 & 104.8 & 125.7 & 0.5 \\
\hline & {$[0.007]$} & {$[0.001]$} & {$[0.000]$} & {$[]$.} & {$[0.000]$} & {$[]$.} & [0.858] \\
\hline \multirow[t]{2}{*}{$H_{0}:$ Disequalization } & 65.4 & 99.4 & 134.4 & 217.4 & 226.0 & 279.8 & 61.3 \\
\hline & {$[0.007]$} & {$[0.001]$} & {$[0.000]$} & {$[]$.} & {$[0.000]$} & {$[]$.} & {$[0.857]$} \\
\hline
\end{tabular}

Note: generalized Lorenz dominance joint tests on ventiles of children earnings under the counterfactual (panel A) and the actual (panel B) settings, along with gap curve dominance at order 2. Gap cures are defined according to the order of groups defined in panel A and B. Covariances are bootstrapped.

is because the QTE associated to groups that are relatively advantaged (both in actual and counterfactual settings) are nowhere larger than the QTE of groups that are always relatively disadvantaged.

In one out of the 45 cases, involving groups D3 and D5, it is not possible to establish the direction of this dominance relation, since both equalization and disequalization cannot be rejected, while this is not the case for equality of gap curves which is rejected. In other six cases involving the comparisons of groups D1 versus D4-D9, the joint dominance test rejects that the gap curve in the counterfactual setting lies either above or below the gap curve in the actual setting. In these situations, there are high chances that gap curves at higher orders are statistically similar and neutrality cannot be rejected.

To complete the equalization of opportunity test, we now apply tests at higher orders of dominance for conditional cdfs and gap curves to a selected number of groups identified by the graphs in figure 2 of the main paper. There, we test ISD2 relations first, by looking at dominance relations between generalized Lorenz dominance across groups in the actual and counterfactual settings. Results are reported in panels A and B of table 1. For most of the groups considered here, it is possible to conclude with Lorenz curves that group D1 is substantially disadvantaged compared to the others. Groups D3 and D5 are also ordered by $G L$ dominance in each setting. In the actual setting, some of the $p$-values cannot be computed. We rely nevertheless on the fact that the estimated tests statistics are generally large to conclude that some of the null hypothesis are rejected by the data.

Panel $\mathrm{C}$ of the table reports the results for the equalization of opportunity tests. The null hypothesis of neutrality of the policy cannot be rejected at $1 \%$ confidence level in 
Table 2: Equalization of opportunity for selected parental earnings deciles groups

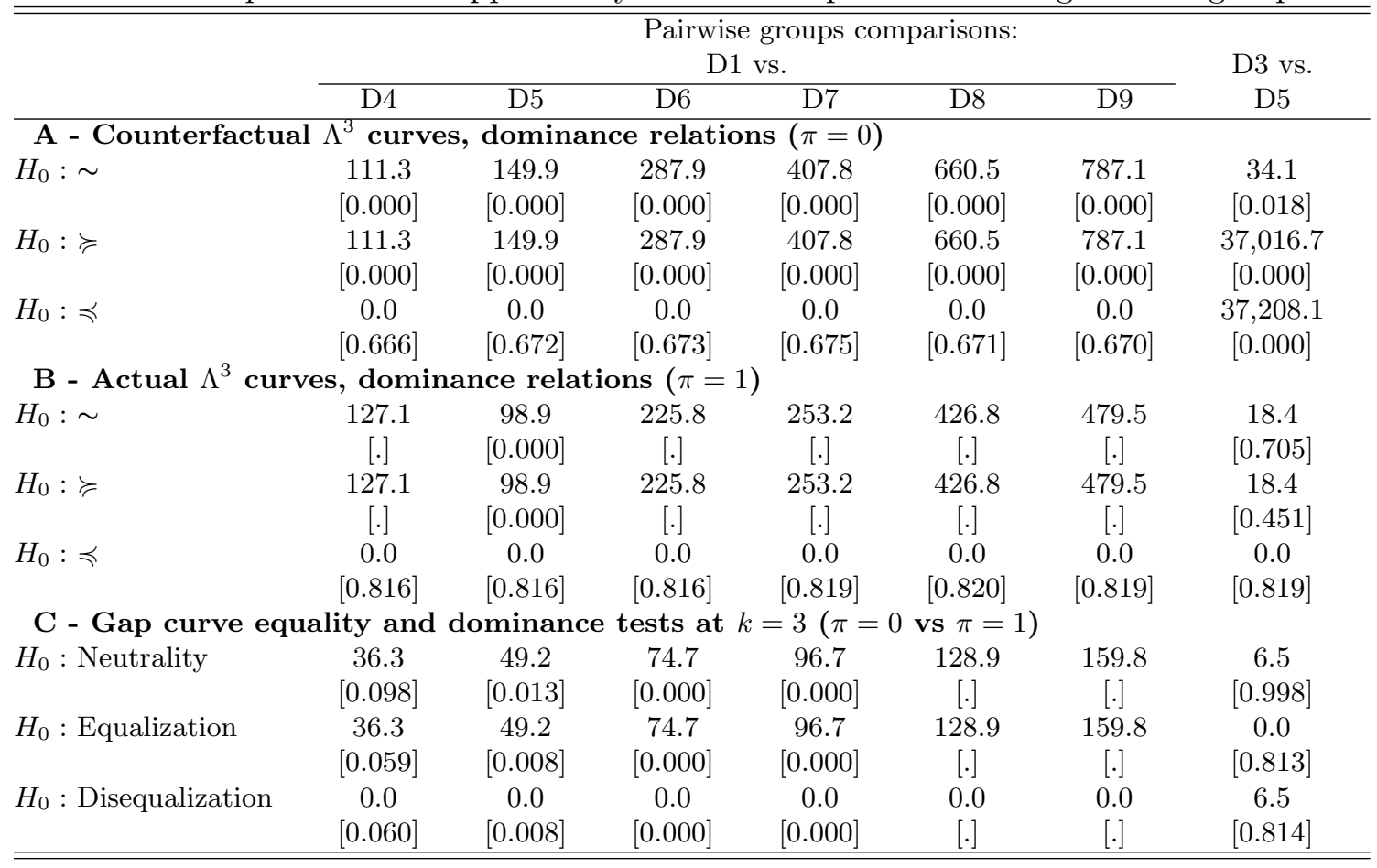

Note: $\Lambda^{3}$ dominance joint tests on ventiles of children earnings under the counterfactual (panel A) and the actual (panel B) settings, along with gap curve dominance at order 3. Gap cures are defined according to the order of groups defined in panel A and B. Covariances are bootstrapped.

comparisons involving groups D1 and D4, while both equalization and disequalization involving groups D1 and the rest are rejected. Equalization and disequalization involving D3 and D4 cannot be rejected at conventional confidence levels, although equality is rejected. Altogether these results indicate that higher order dominance analysis is needed.

The equalization tests for some of the comparisons involving group D1 are not conclusive, while for some of these comparisons it is not possible to obtain reliable measure of the $p$-values associated to joint dominance tests. We conclude that within the set of preferences $\mathcal{R}^{2}$ it is not possible to assess if the gap between groups D1-D5, D1-D6, D1-D7, D1-D8 and D1-D9 has been reduced by the Kindergarten Act.

By further restricting consensus on equalization to the class $\mathcal{R}^{3}$, implemented through ISD3 tests for $\Lambda^{3}$ curves and their associated gap curve, results are more clearcut. The joint tests reported in table 2 allow to conclude in favor of neutrality of the policy with respect to the gap between groups D3 and D5. Furthermore, there is substantial evidence (despite missing $p$-values, the size of test statistic is larger than the rejection level) of disequalization of opportunity between type D1 and the other types (the Wald joint tests for disequalization of the opportunity profiles between group D1 and the rest are all zero). Figure 5 motivates that in large part these results are driven by disequalization of opportunity taking place at the bottom of the group D1 children earnings distributions. 

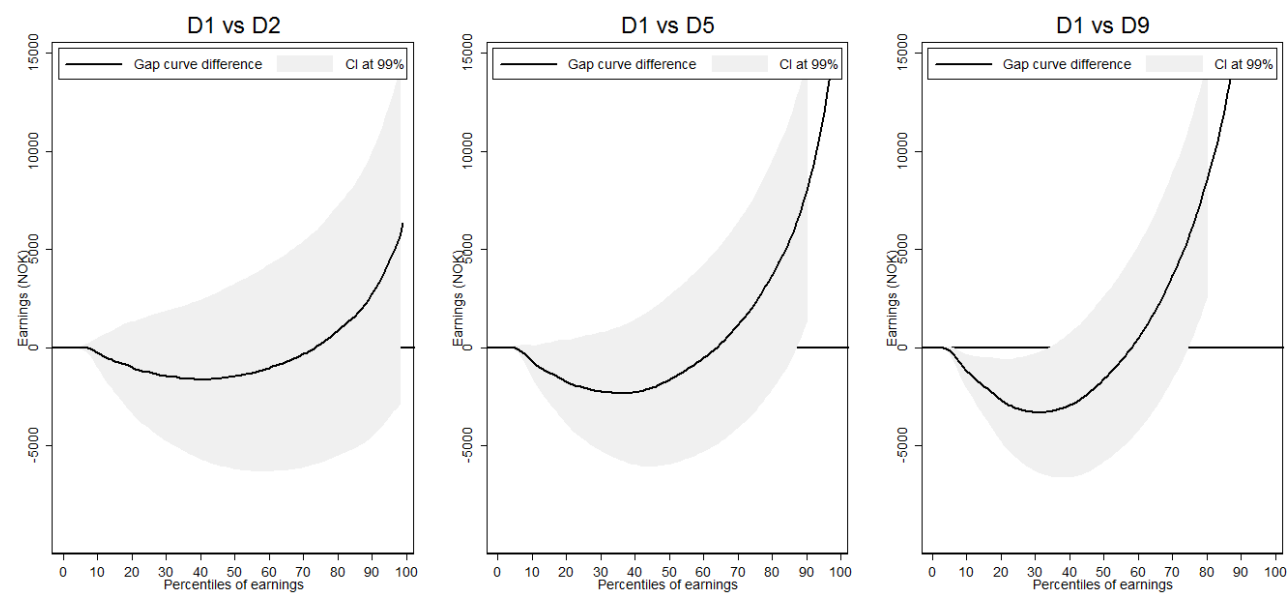

Figure 5: Comparisons of generalized Lorenz curves through gap curves at order 2 for selected pairs of groups

Note: Bootstrapped standard errors.

\section{References}

Aaberge, R., Havnes, T. and Mogstad, M. (2014). A theory for ranking distribution functions. UiO Memo 20/2014.

Andreoli, F. (2017). Robut inference for inverse stochastic dominance, Journal of Business 85 Economic Statistics (forthcoming).

Atkinson, T. B. (1970). On the measurement of inequality, Journal of Economic Theory 2: $244-263$.

Beach, C. M. and Davidson, R. (1983). Distribution-free statistical inference with Lorenz curves and income shares, The Review of Economic Studies 50(4): 723-735.

Dardanoni, V. and Forcina, A. (1999). Inference for Lorenz curve orderings, Econometrics Journal 2: 49-75.

Davidson, R. and Duclos, J.-Y. (2000). Statistical inference for stochastic dominance and for the measurement of poverty and inequality, Econometrica 68(6): 1435-1464.

Gastwirth, J. L. (1971). A general definition of the Lorenz curve, Econometrica 39(6): 1037-1039.

Hadar, J. and Russell, W. R. (1969). Rules for ordering uncertain prospects, The American Economic Review 59(1): 25-34.

Havnes, T. and Mogstad, M. (2011). No child left behind: Subsidized child care and children's long-run outcomes, American Economic Journal: Economic Policy 3: 97129 .

Havnes, T. and Mogstad, M. (2015). Is universal child care leveling the playing field?, Journal of Public Economics 127: 100-114. 
Kodde, D. A. and Palm, F. C. (1986). Wald criteria for jointly testing equality and inequality restrictions, Econometrica 54(5): pp. 1243-1248.

Maccheroni, F., Muliere, P. and Zoli, C. (2005). Inverse stochastic orders and generalized Gini functionals, METRON 63(3): 529-559.

Muliere, P. and Scarsini, M. (1989). A note on stochastic dominance and inequality measures, Journal of Economic Theory 49(2): 314 - 323.

Peragine, V. (2004). Ranking income distributions according to equality of opportunity, Journal of Economic Inequality 2: 11-30.

Roemer, J. E. (2012). On several approaches to equality of opportunity, Economics and Philosophy 28: 165-200.

Roemer, J. E., Aaberge, R., Colombino, U., Fritzell, J., Jenkins, S. P., Lefranc, A., Marx, I., Page, M., Pommer, E., Ruiz-Castillo, J., Segundo, M. J. S., Tranaes, T., Trannoy, A., Wagner, G. G. and Zubiri, I. (2003). To what extent do fiscal regimes equalize opportunities for income acquisition among citizens?, Journal of Public Economics 87(3-4): $539-565$.

Shorrocks, A. F. (1983). Ranking income distributions, Economica 50(197): pp. 3-17.

Zheng, B. (2002). Testing Lorenz curves with non-simple random samples, Econometrica 70(3): 1235-1243. 